\title{
EL CRECIMIENTO ECONÓMICO, LA TEORÍA DE LA POBLACIÓN Y LA FISIOLOGÍA: LA INFLUENCIA DE LOS PROCESOS A LARGO PLAZO EN LA ELABORACIÓN DE LA POLÍTICA ECONÓMICA *
}

ROBERT W. FOGEL **

Universidad de Chicago

La historia económica ha contribuido significativamente a la formulación de la teoria económica. Entre los economistas que han encontrado en la historia una importante fuente de ideas se encuentran Adam Smith, Thomas R. Malthus, Karl Marx, Alfred Marshall, John Maynard Keynes, John R. Hicks, Kenneth J. Arrow, Milton Friedman, Robert M. Solow y Gary S. Becker. Como subrayó S. Kuznets ' ${ }^{1}$ cuando no se ha tomado en consideración la historia, los investigadores que no se han dado cuenta de que sus generalizaciones se basa-

* Este artículo es la conferencia que pronunció Robert W. Fogel en Estocolmo (Suecia) el 9 de diciembre de 1993 en el acto de entrega del Premio Nobel de Economía y se publica aquí con el permiso de la Nobel Foundation.

c) The Nobel Foundation 1993.

** Graduate School of Business, University of Chicago, 1101 East 58th Street, Chicago, Il 60637. Dado que esta conferencia se basa en investigaciones que aún están realizándose, es importante hacer hincapié en que los resultados aqui publicados son provisionales y pueden cam biar conforme se expandan las bases actuales de datos y se refinen los análisis de estos datos. No obstante, creemos que el esbozo general de las nuevas teorias de la mortalidad, la morbilidad y el envejecimiento que están surgiendo probablemente sobrevivirán a las nuevas investigaciones. La investigación publicada en este artículo se ha financiado con ayudas de los National Institutes of Health (No. PO1-AG10120-02), la National Science Foundation (No. SES-9114981) y la Walgreen Foundation. Nos han resultado útiles los comentarios y críticas de Christopher J. Acito, Robert M. Adams, Gary S. Becker, Christine K. Cassel, Katherine A. Chavigny, Dora L. Costa, William J. Darby, Partha Dasgupta, Sidney Davidson, Stanley L. Engerman, Phyllis Eveleth, Enid M. Fogel, Milton Friedman, Victor R. Fuchs, Zvi Griliches, Robin M. Hogarth, Susan E. Jones, John M. Kim, Peter Laslett, Lionel W. McKenzie, Reynaldo Martorell, Douglass C. North, S. Jay Olshansky, Clayne L. Pope, Samuel H. Preston, Irwin Rosenberg, Roger A. Schofield, Nevin S. Scrimshaw, Robert M. Solow, Richard H. Steckel, David Surdam, Richard Suzman, James M. Tanner, Peter Temin, James Trussell, James W. Vaupel, Hans T. Waaler, Larry T. Wimmer y E. A. Wrigley.

1 Simon Kuznets (1941) 
ban en circunstancias transitorias a menudo han entendido mal los problemas económicos del momento. En ningún caso es más importante la necesidad de reconocer el papel que desempeña la dinámica a largo plazo que en algunas cuestiones actuales tan acuciantes como la asistencia médica, la política de pensiones y la política de desarrollo.

En esta conferencia esbozamos una teoría del descenso secular de la morbilidad y la mortalidad que tiene en cuenta los cambios que se han introducido en el funcionamiento fisiológico desde 1700. La sinergia entre las mejoras tecnológicas y las fisiológicas ha generado un tipo de evolución humana, biológica, aunque no genética, rápida, transmitida culturalmente y no necesariamente estable que prosigue tanto en los países de la OCDE como en los países en vías de desarrollo. Definimos los aspectos termodinámicos y fisiológicos del crecimiento económico y se valora su influencia en las tasas de crecimiento. Consideramos las implicaciones de esta teoria para la predicción de la población, la medición de la renta nacional, la demanda de ocio, la política de pensiones y la demanda de asistencia sanitaria.

Hasta la Primera Guerra Mundial no se intentó explicar el descenso secular de la mortalidad de una manera sistemática, debido a que hasta entonces no se sabía con seguridad si estaba descendiendo. Son dos las razones por las que se tardó en reconocer el fenómeno. En primer lugar, hasta el final de las guerras napoleónicas se supo poco sobre las tasas de mortalidad. Antes de 1815 apenas existía una docena de tablas de mortalidad realizadas por varios pioneros de la demografía, las cuales no mostraban una clara tendencia temporal 2. En segundo lugar, apenas había pruebas en las primeras cuatro tablas de mortalidad inglesas oficiales, que se referian a los años 1831-1880, de que la mortalidad estaba mostrando una tendencia descendente.

En la tercera década del siglo $\mathrm{xx}$, sin embargo, comenzó a ser evidente que los nuevos descensos de las tasas británicas de mortalidad no eran simplemente un fenómeno cíclico. Entre 1871 y 1901, la esperanza de vida británica aumentó en cuatro años. Durante las tres décadas siguientes aumentó en otros 16. También se registraron descensos similares de la mortalidad en otros países europeos.

La caída que experimentaron las tasas de mortalidad durante las primeras décadas del siglo xx supusieron un duro golpe para la teoría malthusiana de la población. Se suponia que las mejoras de la mortalidad eran breves porque dada la presión demográfica a que estaban sometidas las existencias de alimentos y que Malthus habia especificado, la erradicación de las muertes provocadas por

\footnotetext{
2 Louis I. Dublin y Alfred J. Lotka (1936), H. Gille (1949).
} 
una enfermedad sería sustituida por otras causadas por alguna otra enfermedad. Los intentos de conciliar la doctrina malthusiana con el descenso observado de la mortalidad, de modificarla o de sustituirla dieron origen a una abundante y nueva literatura.

\section{EXPLICACIÓN DEL DESCENSO SECULAR DE LA MORTALIDAD}

El deseo de explicar el descenso secular de la mortalidad hizo que las investigaciones tomaran tres rumbos distintos. En primer lugar, se realizó un esfuerzo concertado por desarrollar series temporales de las tasas de mortalidad que se remontaran lo más posible con el fin de averiguar exactamente cuándo comenzó el descenso de la mortalidad. En segundo lugar, se analizaron los datos existentes sobre las tasas de mortalidad para identificar los factores que podrian explicar el descenso, así como para establecer pautas o «leyes» que permitieran predecir la futura evolución de la mortalidad.

En tercer lugar, se realizó un esfuerzo general por averiguar la relación entre las existencias de alimentos y las tasas de mortalidad. Este esfuerzo tenía varios aspectos. Tal vez el más importante fuera la aparición de una ciencia de la nutrición que identificó una serie de enfermedades relacionadas con deficiencias nutritivas específicas y que descubrió la sinergia entre la nutrición y la infección ${ }^{3}$. Otro aspecto fue la aparición del campo de la economía del desarrollo después de la Segunda Guerra Mundial dentro de la campaña organizada para eliminar el enorme abismo que existia entre los paises industrializados y los «paises en vías de desarrollo» en lo que se refería a la renta, la salud y la esperanza de vida. Otro aspecto fue el esfuerzo conjunto de los historiadores económicos y demográficos por estudiar el papel de las crisis de la mortalidad y su relación con las hambrunas de los siglos XVII y XviII.

Hasta la década de 1960, los intentos de reconstruir la tendencia secular de la mortalidad europea se concentraron principalmente en comunidades y parroquias notables. Sin embargo, los avances logrados en las décadas de 1960 y 1970 en el desarrollo de técnicas estadísticas y la notable reducción de los costes de cálculo permitieron elaborar y procesar grandes muestras representativas de todo un pais. La figura 1 muestra los resultados de estos intentos, junto con estadisticas oficiales posteriores a 1830 en el caso de Francia y a 1871 en el de Inglaterra. El análisis de la serie francesa y de la británica reveló que

\footnotetext{
${ }^{3}$ Nevin S. Scrimshaw et al. (1968).
} 


\section{FIGURA 1}

Las tendencias seculares de las tasas de mortalidad en Inglaterra y Francia
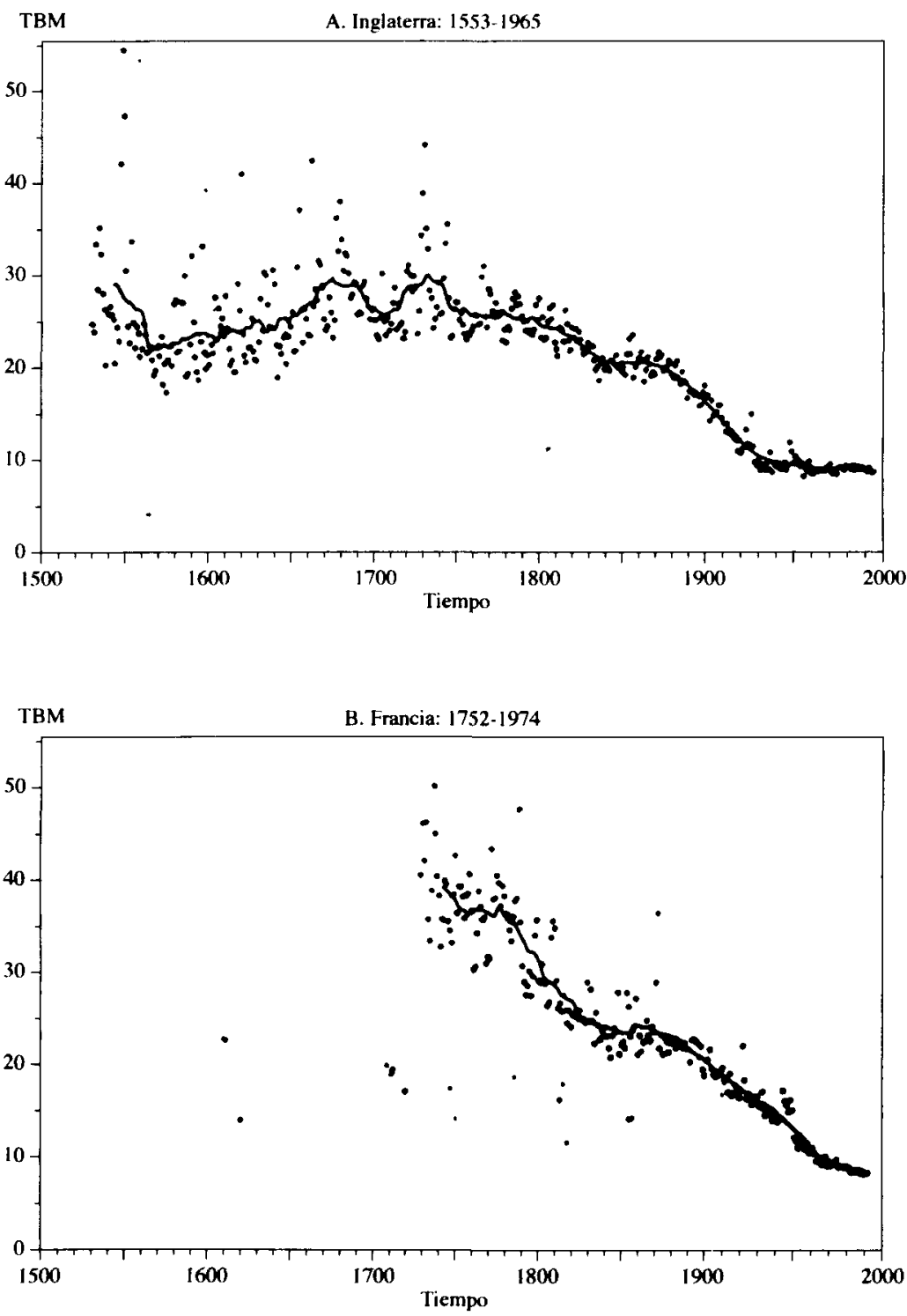

NotA: Los dos gráficos muestran la dispersión de las tasas anuales de mortalidad en torno a una media móvil de 25 años. Véase Fogel y Roderick Flud (1994) para las fuentes y los procedimientos. 
el descenso secular de la mortalidad se produjo en dos oleadas. En el caso inglés, la primera oleada comenzó durante los segundos veinticinco años del siglo XVIII (XVIII-2) y la segunda duró hasta los primeros veinticinco años del siglo XIX, tras lo cual las tasas de mortalidad se estabilizaron durante cincuenta años. El descenso se reanudó durante los cuartos veinticinco años del siglo XIX y ha continuado hasta la actualidad. El caso francés es similar, con la salvedad de que la primera oleada del descenso de la mortalidad se registró alrededor de cincuenta años antes en Francia y su tasa fue más rápida durante esta primera oleada.

Tal vez el aspecto más sorprendente de la figura 1 sea la implicación de que la eliminación de los niveles de mortalidad que representaban situaciones de crisis, relacionadas o no con hambrunas, representaba menos de un 10 por ciento del descenso de las tasas de mortalidad ${ }^{4}$. También se obtuvieron conclusiones similares en estudios de las estadísticas oficiales de Suecia ${ }^{5}$. Al demostrar que estas hambrunas y la mortalidad provocadas por ellas son una cuestión secundaria en la eliminación de las elevadas tasas de mortalidad de los primeros años de la era moderna, estos estudios llevaron a centrar la atención en una cuestión dejada de lado, como es la desnutrición crónica como principal vía a través de la cual la desnutrición contribuyó a las elevadas tasas de mortalidad registradas en el pasado ${ }^{6}$.

\section{LA SINERGIA ENTRE LOS ANÁLISIS BIOMÉDICOS DE LA TENDENCIA SECULAR DE LA DESNUTRICIÓN CRÓNICA Y LOS ECONÓMICOS}

Cuando se utilizan las técnicas biomédicas que se han desarrollado recientemente junto con las económicas, es posible esclarecer el alcance de la desnutrición crónica desde comienzos del siglo XVIII en Europa y Norteamérica, representar y explicar la erradicación de esa desnutrición y examinar la influencia de la mejora de la nutrición en la tendencia secular de la salud y la esperanza de vida, en la productividad del trabajo y en el crecimiento económico. La combinación del modo de análisis económico con el biomédico ha sido sinérgica, ya que ha aportado ideas analíticas que no podrían haberse conseguido basándose meramente en las técnicas de una de las disciplinas.

\footnotetext{
Ronald Lee (1981), E. A. Wrigley y R. S. Schofield (1981), David R. Weir (1982), (1989); Toni Richards (1984), Patrick R. Galloway (1986), Jacques Dupâquier (1989), Fogel (1992b).

5 Tommy Bengtsson y Rolf Ohlsson (1984), (1985); Galloway (1987) (cf. Zvi Eckstein et al. (1984), Gunnar Fridlizius (1984), Alfred Perrenoud (1984), (1991).

6 Cf. Amartya Sen (1981).
} 
La desnutrición puede deberse a una dieta inadecuada o a que las demandas que se hacen a esa dieta (incluido el trabajo y las enfermedades) son tan grandes que producen una desnutrición a pesar de que la ingesta de nutrientes se consideraria adecuada en otras circunstancias ?. Apenas puede haber dudas de que las elevadas tasas de morbilidad existentes en los primeros años de la era moderna habrian causado una desnutrición aunque las dietas hubieran sido adecuadas en calorias, proteínas y otros nutrientes básicos. Sin embargo, las investigaciones recientes indican que en muchos países europeos la producción nacional de alimentos era tan baja antes de mediados del siglo xIX que las clases más pobres estaban abocadas a estar desnutridas en cualquier circunstancia concebible y que las elevadas tasas de morbilidad del periodo no eran meramente una causa de desnutrición sino, indudablemente, en un grado considerable, una consecuencia de unas dietas excesivamente pobres.

\section{A. Contabilidad del coste de la energía y tendencias seculares del tamaño del cuerpo}

Como consecuencia de los estudios de los historiadores agricolas, hoy contamos con estimaciones de la producción agricola británica a intervalos de cincuenta años que se remontan a 1700 . Constituyen la base de los balances nacionales de alimentos que indican la tendencia secular del consumo británico de calorías ${ }^{8}$. Estas fuentes, complementadas con encuestas a los hogares sobre las compras de alimentos, indican que en 1790 el consumo británico medio diario de calorías era del orden de $1.060 \mathrm{kcal}$. per cápita o del orden de 2.700 kcal. por unidad consumidora (equivalentes de varones adultos) ${ }^{9}$. En Francia, Jean-Claude Toutain ha realizado estimaciones a partir de los balances nacionales de alimentos que se remontan a la década anterior a la revolución francesa ${ }^{10}$. Sus estimaciones indican que el consumo diario per cápita de calorias

7 Antes de analizar la desnutrición crónica, es necesario aclarar una confusión terminológica que ha desorientado a algunos investigadores: la distinción entre el término «dieta» o ingestion de alimentos (que representa la nutrición bruta) y el término «desnutrición» (que representa la nutrición neta y que son los nutrientes disponibles para mantener el crecimiento celular). No nos extenderemos aquí en esta distincion; sólo haremos hincapié en que cuando nos refiramos a la nutrición bruta utilizaremos el término «dieta» y que otros términos como «desnutrición», «subnutrición», «nutrición neta» y «situación nutricional» se refieren al equilibrio entre la ingesta de nutrientes (dieta) y lo que se le exige a esa dieta. Véase Fogel y Floud (1994) para una ampliación mayor de esta distinción.

8 J. A. Chartres (1985), B. A. Holderness (1989), R. C. Allen (1994).

9 Carole Shammas (1984), (1990); D. J. Oddy (1990), (cf. Fogel (1987).

10 Jean-Claude Toutain (1971). 
fue de $1.753 \mathrm{kcal}$. en $1781-1790$ y de $1.846 \mathrm{kcal}$. en $1803-1812$. Estas cifras, convertidas en calorías por unidad consumidora, se convierten en $2.290 \mathrm{kcal}$. y 2.410 kcal., respectivamente.

Estas estimaciones implican que los adultos maduros de finales del siglo XVIII debían de ser muy pequeños en comparación con los actuales. Actualmente, el varón de la Unión representativo de 30 a 35 años mide alrededor de 177 centímetros (69,7 pulgadas) y pesa alrededor de 78 kilos (172 libras) ${ }^{11}$. Un varón de esas características requiere diariamente alrededor de $1.794 \mathrm{kcal}$. para el metabolismo basal (la energia necesaria para mantener el cuerpo en funcionamiento mientras está en reposo) y un total de $2.279 \mathrm{kcal}$. para el mantenimiento minimo (las $1.794 \mathrm{kcal}$. necesarias para el metabolismo basal más $485 \mathrm{kcal}$. para la digestión de los alimentos y la higiene vital) ${ }^{12}$. Si los británicos o los franceses hubieran sido tan grandes en el siglo xvIII, casi toda la energía producida por sus existencias de alimentos habría sido necesaria para el mantenimiento y apenas habria quedado para poder trabajar. Para tener la energía necesaria para producir los productos nacionales de estos dos países en torno a 1700 , el varón adulto representativo debió ser bastante bajo y muy delgado.

Esta deducción es confirmada por los datos sobre la estatura y el peso que se han recogido en los paises europeos. El cuadro 1 muestra estimaciones de la estatura final de los varones adultos que llegaron a la madurez entre 1750 y 1875. Indica que durante los siglos XVIII y XIX los europeos eran muy raquíticos en comparación con los actuales (cf. línea 6 del cuadro 1). Las estimaciones del peso de los europeos anteriores a 1860 son mucho más fragmentarias. Las que existen, que son en su mayoría el resultado de deducciones, sugieren que alrededor de 1790 el peso medio de los varones ingleses de 30 a 40 años era del orden de 61 kilos (134 libras), es decir, alrededor de un 20 por ciento menor que el actual. Es posible que la cifra correspondiente a los varones franceses de alrededor de 1790 sólo fuera de unos 50 kilos (alrededor de 110 libras), es decir, alrededor de un tercio menor que la actual.

\section{B. Distribución del consumo de calorías}

La sinergia entre el análisis económico y el biomédico es evidente en los nuevos conocimientos que se obtienen dejando de basarse en la estatura me-

1 U. S. Department of Health and Human Services [USDHHS] (1987)

Quenouille et al. (1951); Organización para la Agricultura y la Alimentación, Universidad de las Naciones Unidas, Organización Mundial de la Salud [FAO/UNU/OMS], (1985). 
dia, el peso medio y el consumo diario medio de nutrientes y utilizando las distribuciones de estas variables. Debido a la falta de tiempo, centraremos la atención aqui en la distribución de las calorías ${ }^{13}$.

\section{CUADRO 1}

Estaturas finales medias estimadas de los varones que llegaron a la madurez entre 1750 y 1875 en seis poblaciones europeas, por periodos de cuartos de siglo

\begin{tabular}{|c|c|c|c|c|c|c|c|}
\hline \multirow{2}{*}{ Fila } & \multirow{2}{*}{$\begin{array}{l}\text { Fecha de } \\
\text { madurez por } \\
\text { siglo y cuarto } \\
\text { de siglo }\end{array}$} & \multicolumn{6}{|c|}{ Estatura (cm.) } \\
\hline & & $\begin{array}{c}\text { Gran } \\
\text { Bretaria }\end{array}$ & Noruega & Suecia & Francia & Dinamarca & Hungria \\
\hline 1 & 18-III & 165,9 & 163,9 & 168,1 & - & - & 168,7 \\
\hline 2 & 18-IV & 167,9 & - & 166,7 & 163,0 & 165,7 & 165,8 \\
\hline 3 & 19.1 & 168,0 & - & 166,7 & 164,3 & 165,4 & 163,9 \\
\hline 4 & 19-II & 171,6 & - & 168,0 & 165,2 & 166,8 & 164,2 \\
\hline 5 & 19-III & 169,3 & 168,6 & 169,5 & 165,6 & 165,3 & - \\
\hline $6 \ldots \ldots$ & 20-III & 175,0 & 178,3 & 177,6 & 172,0 & 176,0 & 170,9 \\
\hline
\end{tabular}

Fuentes: Fogel (1987, cuadro 7) para todos los países salvo Francia. En el caso de Francia, las filas 3.5 se han calculado a partir de M. A. von Meerton (1989), tal como ha sido modificado por Weir (1993), añadiendo $0,9 \mathrm{~cm}$. para tener en cuenta el crecimiento adicional registrado entre los 20 años y la madurez (Benjamin A. Gould, 1869, pp. $104-$ 5) (cf. Gerald C. Friedman, 1982, p. 510 [nota 14]). La cifra de la fila 2 se basa en una extrapolación lineal retrospectiva a 1788 de los datos de Meerton correspondientes a 1815-1836; hemos añadido $0,9 \mathrm{~cm}$. para tener en cuenta el crecimiento adicional registrado entre los 20 años y la madurez. La cifra de la fila 6 procede de Fogel (1987, cuadro 7).

La distribución del consumo calórico es uno de los instrumentos más potentes para saber hasta qué punto son razonables las estimaciones realizadas de las dietas medias. No sólo influyen en las implicaciones de un determinado nivel de consumo calórico para las tasas de morbilidad y mortalidad, sino que también indican si las calorías disponibles para trabajar son coherentes con el nivel de producción agrícola y con la distribución de la población trabajadora entre la agricultura y los demás sectores ${ }^{14}$. Aunque los balances nacionales de alimentos, como los que realizó Toutain ${ }^{15}$ en el caso de Francia

13 Véase el apéndice de Floud y Fogel (1994) para las distribuciones estimadas de la estatura, el peso y el índice de masa corporal (IMC) de Francia alrededor de 1790.

${ }^{14}$ Fogel (1991), Fogel y Floud (1994), cf. Wrigley (1987).

15 Toutain (1971). 
para el periodo 1781-1952 suministran los valores medios del consumo de calorias per cápita, no ofrecen estimaciones de la distribución del consumo de calorías ${ }^{16}$.

Son tres los factores que permiten estimar la distribución del consumo de calorias a partir de la evidencia fragmentaria de que disponen los historiadores. En primer lugar, los estudios que abarcan una amplia variedad de países indican que las distribuciones de las calorias se describen perfectamente por medio de la distribución log-normal. En segundo lugar, la variación de la distribución de las calorías (medida por el coeficiente de variación [s//x] o el coeficiente de Gini [G]) es mucho más limitada que la distribución de la renta. En tercer lugar, cuando se conoce la media de la distribución, el coeficiente de variación (que determina, junto con la media, la distribución) puede estimarse a partir de la información derivada de cualquiera de las dos colas de la distribución. Afortunadamente, incluso en los lugares y los periodos en los que poco se sabe sobre la gente normal, existe una información relativamente abundante sobre los ricos. En el peor de los casos, es la información demográfica, especialmente la tasa de mortalidad, la que limita extraordinariamente la proporción de la población cuyo consumo diario medio de calorías podría haber sido inferior a la tasa metabólica basal (TMB) o mantenimiento mínimo.

El cuadro 2 muestra el nivel excesivamente bajo de capacidad de trabajo que permitían los alimentos existentes en Francia e Inglaterra alrededor de 1790 , incluso después de tener en cuenta las reducidas necesidades para el mantenimiento debido a la pequeña estatura y masa corporal ${ }^{17}$. En Francia, el 10 por ciento inferior de la población trabajadora carecía de la energía necesaria para el trabajo habitual y el 10 por ciento siguiente tenía suficiente energía para realizar menos de tres horas de trabajo ligero diario $(0,52$ horas de trabajo duro). Aunque la situación inglesa era algo mejor, el 3 por ciento inferior de su población trabajadora carecia de la energía necesaria para realizar cualquier trabajo, pero el resto del 20 por ciento inferior tenía suficiente energía para realizar alrededor de 6 horas de trabajo ligero (1,09 horas de trabajo duro) cada día.

El cuadro 2 también señala el problema que plantea el supuesto de que para la población del antiguo régimen era suficiente una ingesta calórica dia-

16 En principio, es posible realizar distribuciones de las calorias por tamaño a partir de las encuestas de consumo de los hogares. Como la mayoria de estas encuestas se basaban en el siglo XIX en las clases más bajas, para utilizarlas es necesario saber de qué centiles de la distribución calórica nacional o de la distribución de la renta nacional procedían los hogares encuestados.

17 cf. Herman Freudenberger y Gaylord Cummins (1976). 
ria de $2.000 \mathrm{kcal}$. per cápita en promedio $(2.600 \mathrm{kcal}$. por unidad consumidora). Ese nivel medio de consumo se encuentra entre los niveles de los franceses y de los ingleses alrededor de 1790. En las poblaciones cuyo nivel de consumo medio era tan bajo, el 20 por ciento inferior subsistía con una dieta tan pobre que quedaba excluido, de hecho, de la población trabajadora; muchos de ellos carecian de la energía necesaria incluso para deambular unas cuantas horas. Éste parece que es el principal factor que explica por qué los mendigos representaban nada menos que una quinta parte de la población en el antiguo régimen ${ }^{18}$. Incluso la mayoría de las personas pertenecientes al 80 por ciento superior de la distribución calórica estaban tan raquíticas (su estatura era inferior a los niveles de la Unión) y delgadas (su peso era inferior a los niveles de la Unión) que corrían muchos más riesgos de padecer enfermedades crónicas y de morir prematuramente (véase el siguiente apartado).

\section{CUADRO 2}

Comparación de las distribuciones francesa e inglesa probables del consumo diario de kilocalorias por unidad consumidora hacia finales del siglo XVIII

\begin{tabular}{|c|c|c|c|c|}
\hline \multirow[b]{2}{*}{ Decil } & \multicolumn{2}{|c|}{ Francia alrededor de 1785} & \multicolumn{2}{|c|}{ Inglaterra alrededor de 1790} \\
\hline & $\begin{array}{c}\text { Consumo } \\
\text { diario de kcal. }\end{array}$ & $\begin{array}{l}\text { Porcentaje } \\
\text { acumulado }\end{array}$ & $\begin{array}{c}\text { Consumo } \\
\text { diario de kcal. }\end{array}$ & $\begin{array}{l}\text { Porcentaje } \\
\text { acumulado }\end{array}$ \\
\hline Superior & 3.672 & 100 & 4.329 & 100 \\
\hline Noveno $\ldots \ldots \ldots \ldots \ldots \ldots$ & 2.981 & 84 & 3.514 & 84 \\
\hline Octavo $\ldots \ldots \ldots \ldots \ldots \ldots \ldots$ & 2.676 & 71 & 3.155 & 71 \\
\hline Séptimo .... & 2.457 & 59 & 2.897 & 59 \\
\hline Sexto $\ldots \ldots \ldots$ & 2.276 & 48 & 2.684 & 48 \\
\hline Quinto $\ldots \ldots \ldots \ldots \ldots \ldots \ldots$ & 2.114 & 38 & 2.492 & 38 \\
\hline$\ldots \ldots \ldots \ldots \ldots$ & 1.958 & 29 & 2.309 & 29 \\
\hline$\ldots \ldots \ldots \ldots \ldots$ & 1.798 & 21 & 2.120 & 21 \\
\hline$\ldots \ldots \ldots \ldots \ldots \ldots$ & 1.614 & 13 & 1.903 & 13 \\
\hline Primero $\ldots \ldots \ldots \ldots \ldots \ldots \ldots$ & 1.310 & 6 & 1.545 & 6 \\
\hline$\ldots \ldots \ldots \ldots$ & 2.290 & & 2.700 & \\
\hline$s / \bar{x}:$ & 0,3 & & 0,3 & \\
\hline
\end{tabular}

FUENTES: Véase Fogel (1993b), especialmente el cuadro 4 y el apéndice.

18 Pierre Goubert (1973), Carlo M. Cipolla (1980), Peter Laslett (1984). 


\section{CURVAS Y SUPERFICIES DE WAALER: UN NUEVO INSTRUMENTO ANALÍTICO}

Los extensos estudios clínicos y epidemiológicos que se han realizado en las dos últimas décadas han demostrado que la estatura correspondiente a determinadas edades, el peso correspondiente a determinadas edades y el peso por unidad de estatura (índice de masa corporal o IMC) son variables que predicen eficazmente el riesgo de morbilidad y mortalidad. Hasta hace poco, la mayoría de los estudios centraban la atención en los niños de menos de 5 años, utilizando uno o más de los indicadores antropométricos correspondientes a estas edades para valorar el riesgo de morbilidad y mortalidad existente en las edades tempranas, y fue en estas edades en las que inicialmente se demostró con mayor firmeza la importancia de las medidas antropométricas ${ }^{19}$. Sin embargo, en los últimos años se ha acumulado una cantidad considerable de datos que sugieren que la estatura correspondiente al momento de llegada a la edad adulta también es una variable importante para predecir la probabilidad de fallecer y de contraer enfermedades crónicas en las edades intermedias y más avanzadas ${ }^{20}$. El IMC tiene las mismas propiedades predictivas ${ }^{21}$

La estatura y el IMC miden aspectos diferentes de la desnutrición y la salud. La estatura no es una medida bruta de la nutrición sino una medida neta. Por otra parte, aunque las variaciones que experimenta la estatura durante los años de crecimiento son sensibles a los niveles de nutrición existentes en ese momento, la estatura final media refleja la experiencia nutricional pasada acumulada por los individuos durante sus años de crecimiento, incluido el periodo fetal. Por lo tanto, cuando se utiliza la estatura final para explicar las diferencias entre las tasas de mortalidad de los adultos, ésta no revela la influencia de los niveles de nutrición de los adultos en las tasas de mortalidad de los adultos, sino de los niveles de nutrición de la infancia, la niñez y la adolescencia en las tasas de mortalidad de los adultos. En cambio, un índice de peso por unidad de estatura refleja principalmente el estado nutritivo correspondiente a cada momento. También es una medida neta en el sentido de que refleja el equilibrio entre la ingesta correspondiente a cada momento y lo que se exige de ella.

19 Alfred Sommer y Matthew S. Lowenstein (1975), Lincoln C. Chen et al. (1980), W. Z. Billewicz e I. A. MacGregor (1982), Arnfried Kelmann et al. (1983), R. Martorell (1985).

20 M. G. Marmot et al. (1984), Hans Waaler (1984), A. Meredith John (1988), Dora L. Costa (1993), John M. Kim (1993).

21 Peter F. Heywood (1983), Waaler (1984), Martorell (1985), P. Payne (1992), S. R. Osmani (1992) (cf. T. N. Srinivasan, 1992). 


\section{A. La relación entre el tamaño del cuerpo y el riesgo de morir en las edades intermedias y avanzadas}

Algunos estudios recientes han demostrado el poder predictivo de la estatura y el IMC con respecto a la morbilidad y la mortalidad en las edades más avanzadas. Las figuras 2 y 3 resumen los resultados de dos de estos estudios. La parte A de la 2 reproduce un gráfico de Waaler ${ }^{22}$. Muestra que los varones noruegos bajos de 40-59 años en situación de riesgo entre 1963 y 1979 tenían muchas más probabilidades de morir que los altos. De hecho, el riesgo de mortalidad de los varones que median $165 \mathrm{~cm}$. (65 pulgadas) era, en promedio, un 75 por ciento mayor que el de los varones que median $182,5 \mathrm{~cm} .(71,9$ pulgadas). La parte $\mathrm{B}$ muestra que la estatura también es un indicador importante para predecir la probabilidad relativa de que los varones de 23-49 años fueran rechazados por el ejército de la Unión en 1861-1865 por padecer enfermedades crónicas. A pesar de la existencia de grandes diferencias entre las etnias, las circunstancias del entorno, la gama y variedad de enfermedades y la época, las relaciones funcionales entre la estatura y el riesgo relativo son sorprendentemente similares en los dos casos.

Waaler ${ }^{23}$ también ha estudiado la relación existente en Noruega entre el IMC y el riesgo de morir en una muestra de 1,7 millones de personas. La figura 3 muestra las curvas que resumen sus resultados correspondientes tanto a los varones como a las mujeres. Aunque los valores observados del IMC $(\mathrm{kg} / \mathrm{m}$ 2) iban desde 17 hasta 39 , más del 80 por ciento de los varones de más de 40 años de edad tenía un IMC situado en el intervalo 21-29. Dentro del intervalo $22-28$, la curva es relativamente plana y el riesgo relativo de mortalidad se mantiene en torno a 1 . Sin embargo, cuando el IMC es menor de 22 y mayor de 28 , el riesgo de morir aumenta bastante acusadamente a medida que el IMC se aleja de su valor medio. Debe señalarse que las curvas del IMC son mucho más simétricas que las curvas de la estatura de la figura 2, lo que indica que los IBM altos son tan arriesgados como los bajos.

Aunque las figuras 2 y 3 son reveladoras, no son suficientes para aportar luz al debate sobre si un raquitismo moderado es perjudicial para la salud cuando el peso por unidad de estatura es adecuado, ya que la figura 2 no tiene en cuenta el peso y la 3 sólo tiene en cuenta, en parte, la estatura ${ }^{24}$. Para llegar a la cuestión de "pequeño pero sano", es necesaria una superficie de isomortalidad que relacione el riesgo de morir tanto con la estatura como con el peso

\footnotetext{
22 Waaler (1984).

23 Waaler (1984).

24 Fogel (1987), Fogel y Floud (1994).
} 


\section{FIGURA 2}

\section{Comparación de la relación entre la estatura corporal y el riesgo relativo de dos poblaciones}

A. Riesgo relativo de mortalidad de los varones noruegos de 40-59 años, entre 1963 y 1979

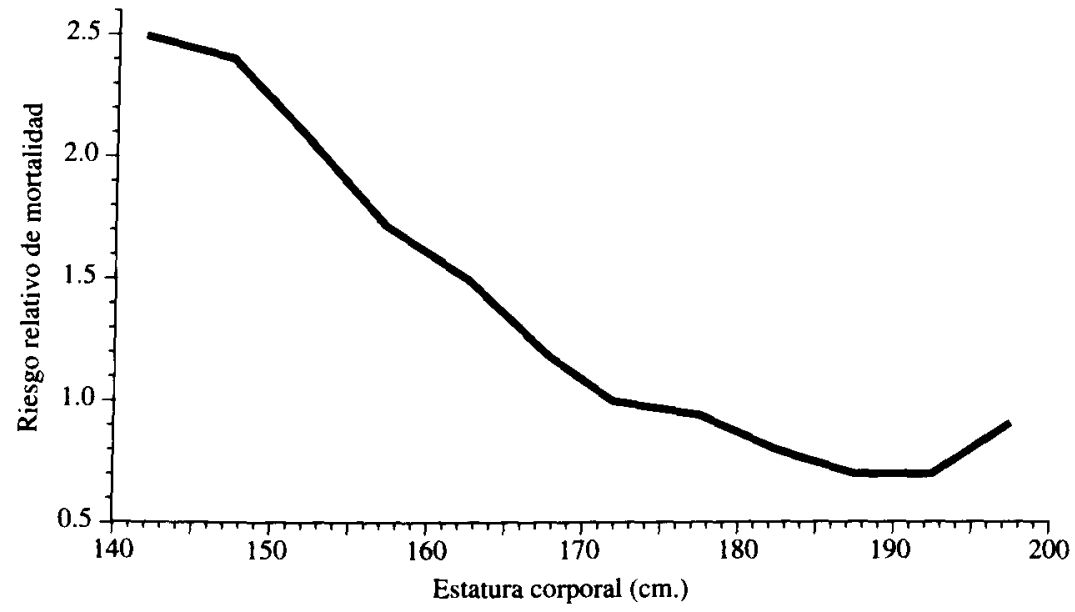

B. Tasas relativas de rechazos provocados por enfermedades crónicas de una muestra de 4.245 varones de 23-49 años, examinados por el ejército de la Unión
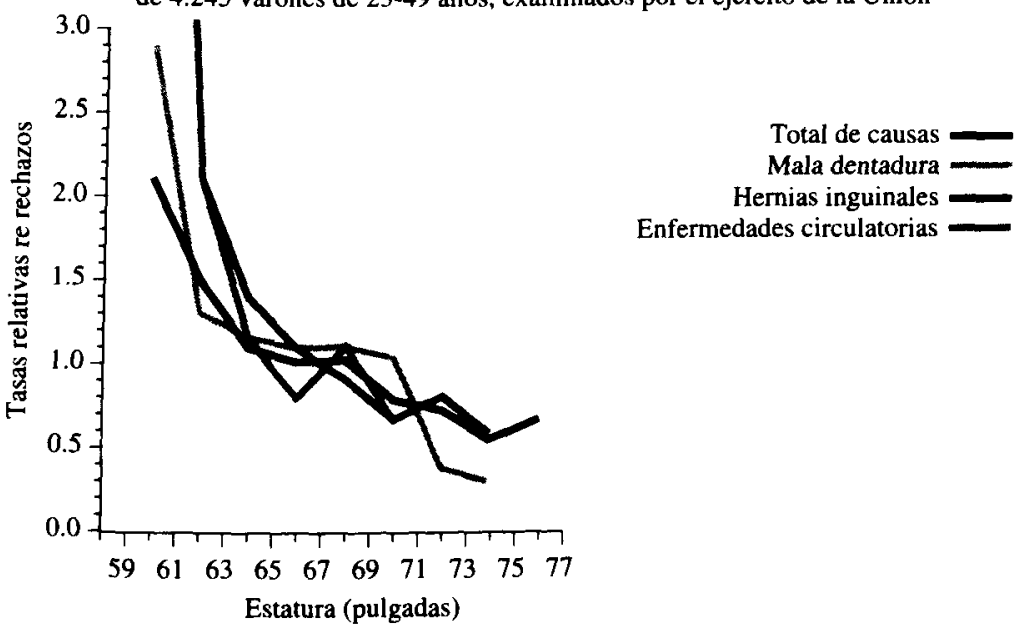

FUENTES: para la parte A, Waaler (1984); para la parte B, Fogel (1993b). 


\section{FIGURA 3}

Relación entre el IMC y el riesgo previsto de los adultos noruegos de 50-60 años en situación de riesgo, entre 1963 y 1979

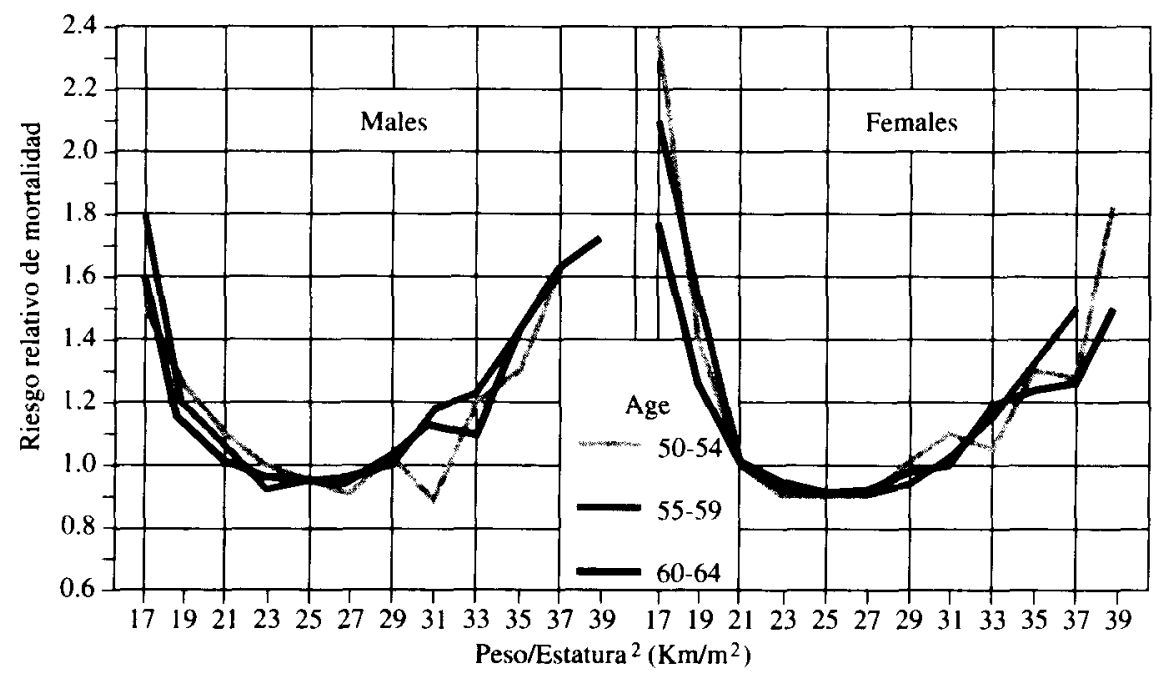

Fuente: Waaler (1984).

simultaneamente. Esa superficie, presentada en la figura 4, se ha ajustado a los datos de Waaler mediante un procedimiento descrito en otro artículo ${ }^{25}$. El mapa de curvas isomortalidad aparece atravesado por lineas que indican el lugar geométrico de cada IMC entre 16 y 34 y una curva que indica los pesos que minimizan el riesgo correspondiente a cada estatura.

La figura 4 muestra que incluso cuando se mantiene el peso del cuerpo en la cifra que la figura 3 indica que es un nivel «ideal» ( $\mathrm{IMC}=25)$, los hombres bajos corren un riesgo mucho mayor de morir que los hombres altos. Así, por ejemplo, un hombre adulto que tenga un IMC de 25 y mida $164 \mathrm{~cm}$. corre un riesgo de morir un 55 por ciento mayor que un hombre que mida $183 \mathrm{~cm}$. y que tenga un IMC de 25. La figura 4 también muestra que el IMC «ideal» (el IMC que minimiza el riesgo de morir) varía con la estatura. Un IMC de 25 es «ideal» para los varones que midan alrededor de $176 \mathrm{~cm}$., pero para los altos (para los que midan más de $183 \mathrm{~cm}$.) el IMC «ideal» se encuentra entre 22 y 24 , mientras que para los bajos (menos de $168 \mathrm{~cm}$.) el IMC «ideal» es del orden de 26 .

25 Fogel (1993b). 


\section{FIGURA 4}

Curvas isomortalidad del riesgo relativo de la estatura y el peso de los varones noruegos de 50-64 años y representación de los valores estimados de la estatura $y$ el peso franceses en cuatro fechas

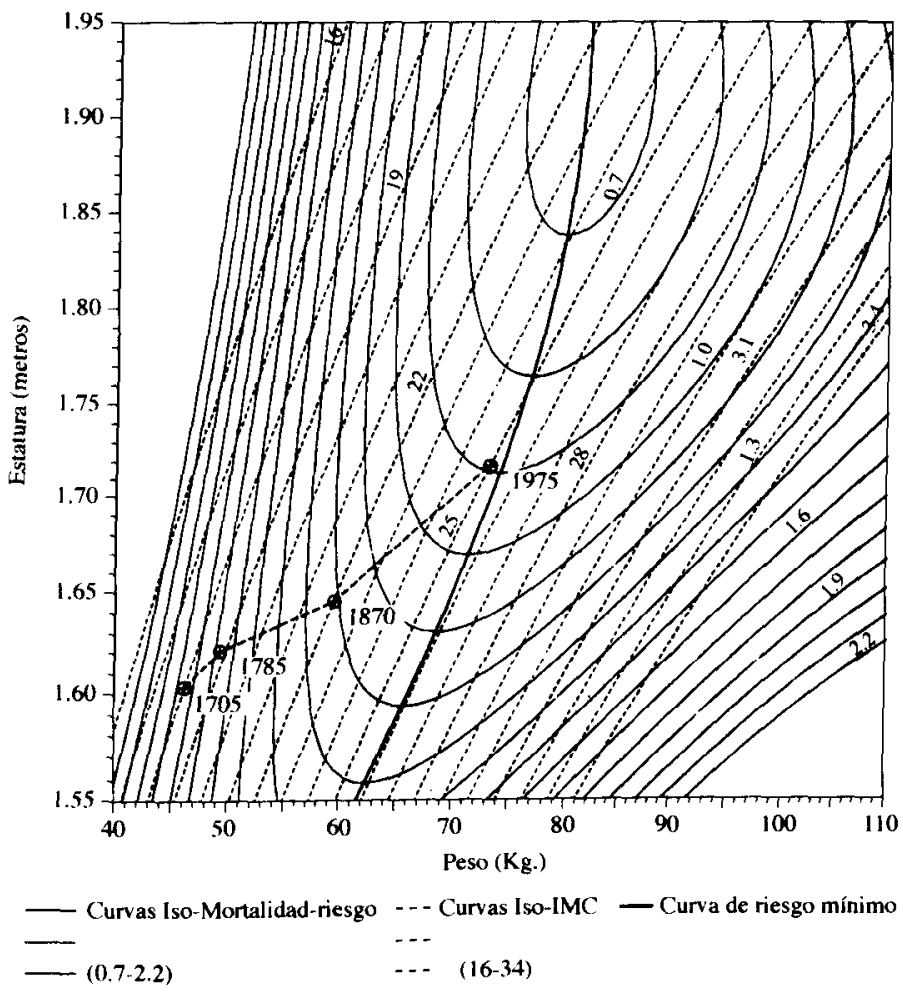

B. La utilización de las superficies de Waaler para explicar el descenso secular de la mortalidad

En la figura 4 hemos superpuesto algunas estimaciones aproximadas de las estaturas y los pesos de Francia correspondientes a cuatro fechas. En 1705, las existencias de alimentos per cápita eran menores en Francia que en Gran Bretaña, por lo que la masa corporal media probablemente era incluso menor que en Gran Bretaña. Alrededor de 1705, los franceses probablemente alcanzaron el equilibrio con sus existencias de alimentos en una estatura media del orden 
de $161 \mathrm{~cm}$. y un IMC de alrededor de 18. En los 270 años siguientes, las existencias de alimentos aumentaron lo suficientemente deprisa para permitir que aumentaran tanto la estatura como el peso de los varones adultos. La figura 4 indica que hasta 1870 son los factores relacionados con el aumento del IMC los que explican la mayor parte de la reducción predicha del riesgo de mortalidad. A partir de ese año, los factores relacionados con el aumento de la estatura explican la mayor parte del descenso predicho de la mortalidad. La figura 4 también implica que aunque estos factores explican conjuntamente alrededor de un 90 por ciento del descenso real de las tasas de mortalidad francesas registrado en el periodo comprendido entre 1785 y 1870 , sólo explican alrededor de un 50 por ciento del descenso real de las tasas de mortalidad registrado durante el siglo pasado. Los aumentos del tamaño del cuerpo y los factores relacionados con él continuaron influyendo significativamente en el aumento de la esperanza de vida de las personas cuya situación nutritiva era relativamente buena, pero durante el siglo pasado cobraron cada vez más importancia otros factores distintos de los relacionados con la estatura y el IMC.

El análisis de este apartado indica el carácter engañoso del concepto de subsistencia tal como lo utilizó inicialmente Malthus y tal como sigue utilizándose ampliamente hoy. La subsistencia no se halla al borde de un precipicio nutritivo, más allá del cual se encuentra el desastre demográfico. La evidencia esbozada en este artículo implica que en lugar de un nivel de subsistencia, hay numerosos niveles en los que una población y unas existencias de alimentos pueden estar en equilibrio, en el sentido de que pueden mantenerse indefinidamente. Sin embargo, algunos niveles tendrán personas más pequeñas y una mayor mortalidad «normal» (sin llegar a ser crítica) que otros 26 .

26 Por otra parte, con una población y una tecnologia dadas, las variaciones de la distribución del trabajo entre la agricultura y otros sectores pueden provocar cambios en el tamaño del cuerpo y en la mortalidad. En una economía del antiguo régimen, cuanto menor es la proporción de la población activa que trabaja en la agricultura, ceteris paribus, menor es la proporción de la producción de calorías que puede dedicarse al mantenimiento mínimo. El razonamiento en que se basa esta afirmación es el siguiente. Supongamos que un trabajador agrícola se alimenta a si mismo y a tres personas más que no trabajan en la agricultura. Por lo tanto, un desplazamiento de un 1 por ciento de los trabajadores agricolas a sectores no agricolas reduciría las existencias per cápita de alimentos de que puede disponer el mayor sector no agricola alrededor de 1,33 por ciento. Si el mantenimiento mínimo representa un 75 por ciento del consumo de calorías y si las calorías per cápita reservadas para el trabajo permanecen constantes en el sector no agrícola, las calorías disponibles para el mantenimiento minimo en ese sector disminuirian alrededor de un 1,8 por ciento (suponiendo que la producción y el consumo per cápita del sector agrícola no varian). 


\section{La importancia de las superficies de Waaler para predecir las tendencias de las enfermedades crónicas}

Una mala complexión corporal aumentaba la vulnerabilidad a las enfermedades, no sólo a las enfermedades contagiosas sino también a las enfermedades crónicas. Esta cuestión está implícita en la figura 2, que muestra que las enfermedades crónicas eran mucho más frecuentes en los varones jóvenes y bajos en la década de 1860 que en los altos. La figura 5 muestra que existe la misma relación entre la mala salud y la estatura en los varones analizados por las U. S. National Health Interview Surveys (NHIS) que abarcan el periodo 1985-1988. El raquitismo durante las edades de desarrollo estaba muy extendido y aumentaba la probabilidad de padecer enfermedades crónicas en las edades intermedias y avanzadas.

Los varones de la Unión nacidos durante los segundos veinticinco años del siglo XIX no sólo estaban raquiticos desde el punto de vista actual, sino que su IMC en las edades adultas era alrededor de un 15 por ciento menor

\section{FIGURA 5}

Relación entre la estatura y el riesgo relativo de mala salud en los excombatientes de las NHIS de 40-59 años

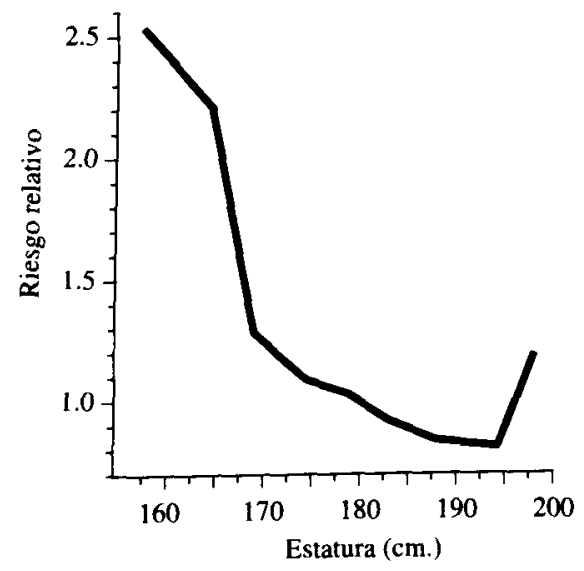

Fuente: Fogel et al. (1993). 
que el nivel de la Unión actual ${ }^{27}$. La implicación de la conjunción del raquitismo y un bajo IMC se muestra en la figura 6, que presenta una superficie de Waaler relativa a la morbilidad estimada por Kim (1993) a partir de datos de las NHIS de 1985-1988.

\section{FIGURA 6}

Mejora de la salud predicha por la superficie de salud de las NHIS de 1985-1988

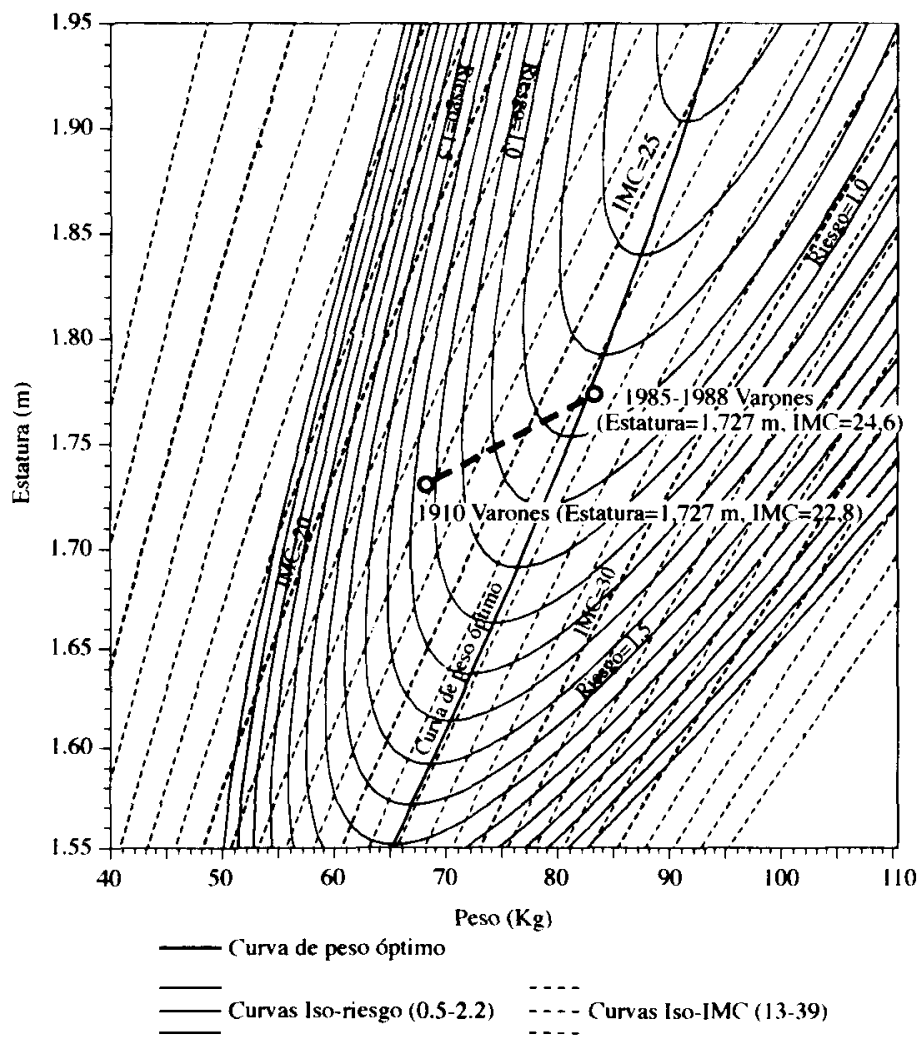

Nota: Todos los riesgos se han medido en relación con el riesgo medio de morbilidad (calculado para todas las estaturas y los pesos) de los varones blancos que tenían entre 45 y 64 años en las NHIS de 1985.1988.

FuENTE: Kim (1993).

\footnotetext{
27 Fogel et al. (1993).
} 
La superficie de Waaler correspondiente al riesgo que representan las enfermedades crónicas de la figura 6 es similar, pero no idéntica, a la superficie noruega correspondiente a la mortalidad (véase la figura 4). Las curvas isomorbilidad correspondientes a una mala salud son más inclinadas que las curvas isomortalidad a medida que nos alejamos en cualquiera de las dos direcciones de la curva óptima de peso. Por otra parte, la curva óptima de peso de la figura 6 normalmente se encuentra alrededor de una curva iso-IMC a la derecha de la curva óptima de peso calculada a partir de los datos noruegos sobre la mortalidad. Por consiguiente, tanto los datos noruegos sobre la mor. talidad como los datos de la Unión sobre la salud indican que en el caso de los varones que miden entre 1,60 y 1,65 metros el IMC óptimo se encuentra entre 25 y 27 . Este nivel es superior al actual que recomienda FAO/WHO/ UNU, que se encuentra en los intervalos más bajos de sobrepeso según dicha norma ${ }^{28}$.

La figura 6 también presenta las coordenadas de estatura e IMC de los excombatientes del ejército de la Unión que tenían 65 años o más en 1910 y de los excombatientes (principalmente de la Segunda Guerra Mundial) que tenían esa mismas edades en 1985-1988. Estas coordenadas predicen una disminución de la incidencia de las enfermedades crónicas en las dos cohortes del orden del 35 por ciento. Alrededor de un 61 por ciento de la disminución predicha de la mala salud se debe a factores relacionados con el aumento del IMC y el resto se debe a factores relacionados con un aumento de la estatura.

La disminución de la incidencia de las enfermedades crónicas que predice la figura 6 se acerca bastante a lo que ocurrió realmente. El cuadro 3 compara el predominio de las enfermedades crónicas en los varones del ejército de la Unión de 65 años o más en 1910 con dos encuestas realizadas a excombatientes que tenian esas mismas edades en la década de 1980. Ese cuadro indica que las enfermedades de corazón eran 2,9 veces más frecuentes, las enfermedades musculares y óseas y las enfermedades respiratorias eran 1,6 veces más frecuentes y las enfermedades digestivas eran 4,7 veces más frecuentes en los excombatientes que tenían 65 años o más en 1910 que en 1985-1988. Durante las 7,6 décadas que separan a estos dos grupos, la incidencia de las enfermedades de corazón en los ancianos disminuyó a una tasa de 12,8 por ciento por década, mientras que las enfermedades musculares y óseas y respiratorias disminuyeron ambas a una tasa de 5,9 por ciento por década.

${ }^{28} \mathrm{FAO} / \mathrm{WHO} / \mathrm{UNU}(1985)$. 


\section{CUADRO 3}

Comparación de la incidencia de las enfermedades crónicas en los excombatientes del ejército de la Unión en 1910, excombatientes en 1983 (que declararon síbabian tenido alguna vez enfermedades crónicas) y los excombatientes de las NHIS de 1985-1988 (que declararon si babian tenido determinadas enfermedades crónicas durante los 12 meses anteriores), 65 años o más, porcentajes

\begin{tabular}{|c|c|c|c|c|}
\hline Enfermedad & $\begin{array}{l}\text { Excombatientes } \\
\text { del ejército de } \\
\text { la Unión en } 1910\end{array}$ & $\begin{array}{l}\text { Excombatientes } \\
\quad \text { en } 1983\end{array}$ & $\begin{array}{c}\text { Excombatientes } \\
\text { en } 1983 \text { ajustados } \\
\text { para tener en } \\
\text { cuenta la edad }\end{array}$ & $\begin{array}{c}\text { Excombatientes } \\
\text { de las NHIS } \\
\text { de } 1985-88\end{array}$ \\
\hline Musculares y óseas & 67,7 & 47,9 & 47,2 & 42,5 \\
\hline Digestivas ..... & 84,0 & 49,0 & 48,9 & 18,0 \\
\hline Hernia ................ & 34,5 & 27,3 & 26,7 & 6,6 \\
\hline Diarrea............ & 31,9 & 3,7 & 4,2 & 1,4 \\
\hline Genito-urinarias & 27,3 & 36,3 & 32,3 & 8,9 \\
\hline $\begin{array}{l}\text { Del sistema nervioso cen- } \\
\text { tral, endocrinas, metabó- }\end{array}$ & & & & \\
\hline licas o sanguineas ....... & 24,2 & 29,9 & 29,1 & 12,6 \\
\hline Circulatorias $^{*} \ldots \ldots \ldots$. & 90,1 & 42,9 & 39,9 & 40,0 \\
\hline Corazón ................ & 76,0 & 38,5 & 39,9 & 26,6 \\
\hline Venas varicosas ........ & 38,5 & 8,7 & 8,3 & 5,3 \\
\hline Hemorroides ${ }^{b}$. & 44,4 & & & 7,2 \\
\hline Respiratorias ............. & 42,2 & 29,8 & 28,1 & 26,5 \\
\hline
\end{tabular}

- Por lo que se refiere a los excombatientes en 1983, la incidencia de todos los tipos de enfermedades circulatorias se subestima debido a que el número de hemorroides declaradas es menor de lo real.

b La variable que indica si el excombatiente en 1983 tuvo alguna vez hemorroides no es fiable.

Notas: Las tasas correspondientes a los excombatientes del ejército de la Unión se basan en exámenes de médicos. Las de los años 80 se basan en declaraciones de los encuestados. La comparación entre las tasas de las NHIS y las de la NHANES II, que proceden de los exámenes de los médicos, indica que la utilización del estado de salud declarado por los encuestados no introdu. ce un sesgo significativo en la comparación. Véase Fogel et al. para un análisis más detallado de los posibles sesgos y sus magnitudes.

FuENTE: Fogel et al. (1993).

Los adultos jóvenes nacidos entre 1822 y 1845 que sobrevivieron a las enfermedades infecciosas mortales de la niñez y la adolescencia no se libraron más de las enfermedades degenerativas que las personas que tienen esas mismas edades hoy, como han sugerido algunos, sino que se vieron más afectados por ellas. Por ejemplo, a los 35-39 años, las tasas de hernias eran más de tres veces más frecuentes en la década de 1860 que en la de 1980. Merece la pena 
señalar especialmente la incidencia mucho mayor de los pies deformes en la década de 1860, anomalia congénita que sugiere que el útero era mucho menos seguro para los que aguardaban el nacimiento que en la actualidad.

Los que sobrevivieron también a las enfermedades de las edades intermedias se vieron más afectados por las enfermedades crónicas degenerativas en las edades avanzadas en la década de 1910 que en la de 1980. Cerca del 74 por ciento de los excombatientes más mayores del ejército de la Unión sufrió tres o más enfermedades crónicas invalidantes, cifra que es muy superior a la tasa correspondiente a los excombatientes de edad avanzada en 198329 . Tal vez sea cierto que había menos personas genéticamente débiles entre las que superaban los 65 años en 1910 que en la actualidad. De ser eso cierto, esa ventaja genética se vio contrarrestada aparentemente por una vida de estrés socioeconómico y biomédico que afectó seriamente a la salud en las edades avanzadas y que redujo acusadamente las esperanzas de vida de los ancianos. Durante la década de 1910 , los ancianos no morian a causa de enfermedades infecciosas que mataban a la inmensa mayoria de sus cohortes a edades relativamente jóvenes, sino principalmente a causa de enfermedades degenerativas que, en la Clasificación Internacional de Enfermedades a dos dígitos, son similares a la distribución de las causas de muerte en la década de 1980, con la salvedad de que las muertes causadas por neoplasmas eran menores y las muertes por tuberculosis eran mayores que en la década de 1980.

Los resultados provisionales sugieren, pues, que las enfermedades crónicas eran mucho más frecuentes durante todo el ciclo vital en el caso de las personas que cumplieron 65 años antes de la Primera Guerra Mundial de lo que sugiere la teoría de la transición epidemiológica. La utilización de la información sobre las causas de muerte para caracterizar la epidemiología del pasado ha llevado a una significativa tergiversación de la distribución del estado de salud de los vivos. También ha fomentado la idea de que la epidemiologia de las enfermedades crónicas es más independiente de la epidemiología de las enfermedades contagiosas de lo que parece actualmente.

\section{FUNDAMENTOS FISIOLOGICOS DE LAS SUPERFICIES Y LAS CURVAS DE WAALER}

¿Cuál es la base de la capacidad de predicción de las superficies y las curvas de Waaler? La respuesta reside, en parte, en el reino de la fisiologia huma-

${ }^{29}$ Fogel et al. (1993). 
na, que se ocupa del funcionamiento de los órganos y de los sistemas orgánicos del cuerpo. Parece que las diferencias de estatura y de peso están relacionadas con las diferencias de composición química de los tejidos que forman estos órganos, de la calidad de la transmisión eléctrica entre las membranas y del funcionamiento del sistema endocrino y otros sistemas vitales.

Las investigaciones sobre esta área están desarrollándose rápidamente y aún están por confirmar algunos de los nuevos resultados. Los mecanismos exactos por los que la desnutrición y los traumas padecidos en el útero o en los primeros años de vida se transforman en disfunciones orgánicas aún no están claros. En lo que todo el mundo está de acuerdo es en que las estructuras básicas de la mayoría de los órganos se establecen pronto y es razonable deducir que los órganos mal desarrollados pueden dejar de funcionar antes que los órganos bien desarrollados. La principal evidencia de que disponemos hasta ahora es estadistica y, a pesar de que existe unanimidad sobre algunas disfunciones específicas, no se dispone de una teoria del envejecimiento celular que sea aceptada por todo el mundo ${ }^{30}$.

Teniendo en cuenta estas salvedades, es útil dividir en tres categorias las investigaciones recientes sobre la conexión entre la desnutrición y el tamaño del cuerpo y la aparición posterior de enfermedades crónicas. La primera categoría está formada por los tipos de desnutrición (incluida la ingestión de sustancias tóxicas) que causan daños fisiológicos permanentes e inmediatamente visibles, como se observa en el deterioro de los sistemas nerviosos de los fetos causado por un consumo excesivo de alcohol o de tabaco por parte de las mujeres embarazadas. Por ejemplo, el alcohol retrasa el crecimiento de los fetos y de los bebés y provoca un defecto septal atrial, microcefalia y otras anomalias congénitas que se denominan colectivamente Sindrome o Sintomas de Alcohol Fetal ${ }^{31}$. Parece que la falta de proteinas en la infancia y en los primeros años de la niñez puede provocar un deterioro permanente de la función del sistema nervioso central ${ }^{32}$. La falta de yodo en el útero y la falta entre moderada y grave de hierro durante la infancia también parece que causan daños neurológicos permanentes ${ }^{33}$.

No todos los daños que se deben a un desarrollo retrasado en el útero o en la infancia causado por la desnutrición se manifiestan inmediatamente. En

30 Cf. J. M. Tanner (1990), (1993).

31 S. L. Robbins et al. (1984).

32 Scrimshaw y J. E. Gordon (1968), Martorell et al. (1990), A. Chávez et al. (1994), cf. J. J. Volpe (1987).

33 Betsy Lozoff et al. (1991), Scrimshaw (1993). 
una reciente serie de estudios, D. J. P. Barker y sus colegas ${ }^{34}$ señalan que algunas enfermedades como las coronarias, la hipertensión, la apoplejía, la diabetes y la tiroiditis autoinmune comienzan en el útero o en la niñez, pero no son evidentes hasta las edades adultas intermedias o las edades más avanzadas. En estos casos, parece que los individuos gozan de buena salud y funcionan bien entre tanto. Sin embargo, la aparición temprana de las enfermedades degenerativas de las edades avanzadas parece ir unida a un desarrollo celular insuficiente durante los primeros años de vida. Algunos de esos casos, pero no todos, están relacionados con la presencia de un bajo peso en el nacimiento. Algunos bebés nacen dentro del intervalo de peso normal, pero engordan menos que la media durante la infancia. En otros casos, los bebés son pequeños en relación con el tamaño de la placenta, bajos en relación con el tamaño de su cabeza o largos pero delgados ${ }^{35}$

Ciertas disfunciones fisiológicas que padecen las personas que sufren desnutrición pueden eliminarse en principio mejorando la ingesta de alimentos, pero a menudo persisten porque persiste la causa de la desnutrición. Si la desnutricion persiste lo suficiente, estas disfunciones pueden volverse irreversibles o fatales. Esta categoría de disfunciones comprende la degradación de la estructura de los tejidos, sobre todo en organos vitales como los pulmones, el corazón y el tracto gastrointestinal. En el caso del sistema respiratorio, por ejemplo, no sólo es menor la masa y la fuerza muscular, sino que también está dañado el mecanismo de ventilación, hay cambios bioquímicos en el tejido conectivo y anomalías electrolíticas. La desnutrición también se ha relacionado con la atrofia de las células mucosas del intestino, la inhibición de la cicatrización de las heridas, el aumento de la probabilidad de sufrir shock traumáticos y sepsis, el mal funcionamiento del sistema endocrino, el aumento de la tendencia a sufrir edemas, la inestabilidad eléctrica que puede provocar arritmias agudas y las enfermedades conjuntas degenerativas ${ }^{36}$.

También es importante el descubrimiento de la relación entre el peso en el nacimiento y la probabilidad de muerte perinatal. Las curvas de la figura 7 tienen forma de $U$, lo que indica que en cada población los bebés que pesan significativamente más de lo óptimo también corren un alto riesgo de morir. Por otra parte, el peso óptimo en el nacimiento de las dos poblaciones en las que las madres eran pequeñas era significativamente menor que el de la población

34 Barker et al. (1989), (1990), (1992); Barker (1991), C. M. Law et al. (1993), D. I. W. Phillips et al. (1993), K. Phipps et al. (1993).

35 Barker (1993) (cf. Tanner (1993).

36 Thomas M. Saba et al. (1983), Juan Idiaquez (1988), G. L. Hill (1990), Janis S. Fisler (1992), cf. Kenneth G. Manton (1993). 


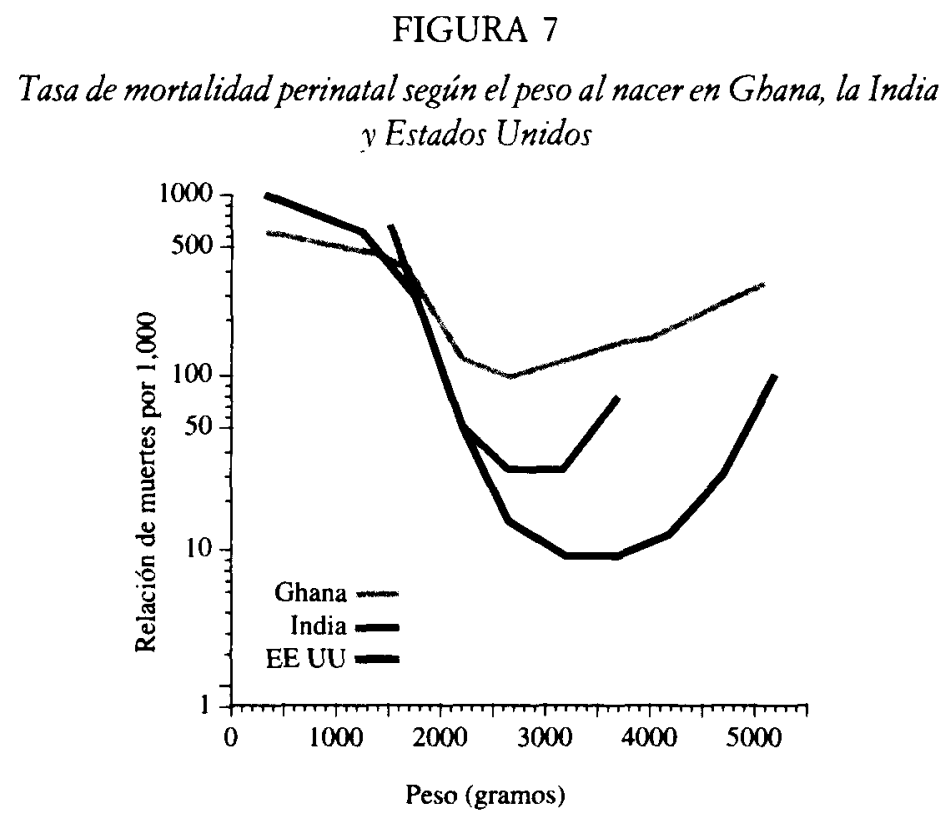

FuENTE: F. E. Hytten e I. Leitch (1971).

americana, en la que las madres eran relativamente grandes. Parece que antes de las intervenciones de alta tecnología, el tamaño de la pelvis de la madre limitaba la tasa a la que podría haber aumentado el tamaño en el nacimiento ( $\mathrm{y}$ quizá la robustez de los órganos vitales del bebé) con la mejora de la nutrición intrauterina. Los bebés que tenian pesos que optimizaban la supervivencia en una población relativamente alta corrían un alto riesgo de morir en las poblaciones en las que las madres eran relativamente bajas, debido al estrés causado en el parto. En otras palabras, había un factor intergeneracional que limitaba la tasa a la que los bebés podian escapar de los efectos de la desnutrición cuando mejoraba la nutrición fetal ${ }^{37}$.

Los recientes resultados fisiológicos también aportan nueva luz sobre la primera fase del descenso secular de la mortalidad. Algunos investigadores han llamado la atención sobre las variaciones de las tasas de mortalidad por edades que pueden indicar un cambio de la relación entre los agentes patógenos y sus anfitriones humanos ${ }^{38}$. Aunque el cambio se ha atribuido a una disminución

${ }^{37}$ Cf. R. K. Chandra (1975).

${ }^{38}$ Fridlizius (1984), Perrenoud (1984). 
de la virulencia de los agentes patógenos, aún no se dispone de suficiente evidencia para valorar esta posibilidad. Sin embargo, las recientes investigaciones fisiológicas resumidas en este apartado sugieren una nueva senda a través de la cual es posible que la relación entre los agentes patógenos y los anfitriones humanos se haya vuelto a favor de los segundos. Además de mejorar el funcionamiento del sistema inmunológico, ha aumentado la capacidad de los órganos vitales para sobrevivir a los ataques de los agentes patógenos al aumentar la elasticidad de los tejidos, incluida la mejora del funcionamiento del sistema nervioso. El proceso podría haber sido sinérgico, ya que es posible que la mejora del funcionamiento del sistema inmunológico haya interactuado con el aumento de la elasticidad de otros órganos vitales ${ }^{39}$. La última posibilidad es coherente con las pautas de descenso de la tasas de mortalidad por edades que se han descubierto hasta ahora en el caso del siglo XviII.

\section{ALGUNAS IMPLICACIONES PARA LA POLITÍCA ACTUAL}

Malthus creía que la desnutrición se manifestaba en acontecimientos excepcionales, es decir, en las hambres periódicas y en la excesiva mortalidad frecuente de los superpobres de su época, que vivian en la miseria y en el vicio. Pensaba que las personas que se encontraban en el punto medio del orden social, los fuertes trabajadores agrícolas o los artesanos de las ciudades, generalmente estaban bien alimentados y sanos y tenían una longevidad normal.

Sin embargo, hoy sabemos que las hambres explican menos del 4 por ciento de la mortalidad prematura de la época de Malthus y que la mortalidad excesiva de los superpobres (el quintil inferior de la sociedad) explica otro sexto de la mortalidad prematura. Alrededor de dos tercios de toda la mortalidad prematura registrada en la época de Malthus procedía del segmento de la sociedad que Malthus consideraba productivo y sano. Sin embargo, desde el punto de vista actual en Gran Bretaña incluso las personas de la mitad superior de la distribución de la renta estaban raquíticas y desgastadas en el siglo XVIII, padecian más enfermedades crónicas en las edades adultas jóvenes e intermedias que hoy y morían 30 años antes que hoy ${ }^{40}$.

39 Esas posibles sinergias ponen en cuestión la proposición de que como parece que en un determinado momento un individuo está bien alimentado, la desnutrición no influye en el resultado de enfermedades como la gripe, la viruela o el tifus (cf. S. R. Duncan et al., 1993). Es más probable que los individuos sucumban a esas infecciones, aunque estén en un determinado momento bien alimentados, debido a que la desnutrición pasada, bien en el útero o posteriormente, ha deteriorado órganos vitales (cf. Scrimshaw et al., 1968).

40) La mortalidad prematura se refiere aqui a las tasas de mortalidad superiores a las de 1980 


\section{A. Implicaciones para los países pobres}

El legado malthusiano se encuentra en tesis como "pequeño pero sano", según la cual los individuos raquíticos o moderadamente desgastados pueden no ser más vulnerables a la falta de salud y a la mortalidad que los que se ajustan al patrón de la Unión. La escasez de datos que abarquen ciclos vitales completos en los países en vías de desarrollo llevó a los investigadores a centrar solamente la atención en los primeros años de vida, buscando interacciones entre los indicadores natales o infantiles del tamaño y los indicadores de la salud y la capacidad de trabajo en la niñez. Esos estudios generalmente tenian en cuenta los efectos del raquitismo y del desgaste excesivamente graves (más de dos desviaciones típicas por debajo de la media), pero no la influencia de los efectos más moderados del tamaño, muchos de los cuales no se manifiestan hasta más tarde ${ }^{41}$.

Sin embargo, la información que presentamos en este artículo indica que el raquitismo y el desgaste en la niñez están muy extendidos y predicen las tasas de enfermedades crónicas correspondientes a los adultos jóvenes y a las personas de edad avanzada. No se ha prestado atención a la mayor incidencia de las enfermedades crónicas invalidantes en los adultos de los países en vías de desarrollo debido a que generalmente no se recoge la información relevante sobre estas enfermedades. Pero la incidencia de la desnutrición en la niñez en los países ricos, tanto hoy como cuando éstos eran mucho más pobres, induce a pensar que también existe una conexión similar en los países en vías de desarrollo.

Las enfermedades crónicas no son sólo la única vía por la que la desnutrición crónica reduce la productividad de la población trabajadora. Cuando las cantidades medias de calorías son tan bajas como en los paises pobres del mundo, las tasas de actividad y los indicadores de la productividad del trabajo están abocados a ser bajos, sobre todo cuando se ajustan las horas de trabajo para tener en cuenta la intensidad del trabajo ${ }^{42}$. En otro lugar he estimado que cuando se ajusta la cantidad de trabajo para tener en cuenta la intensidad

calculadas con la estructura por edades de 1700 (cf. Fogel, 1986, 1992b). Las estimaciones de la tasa global de mortalidad de alrededor de 1790 proceden de Wrigley y Schofield (1981). Las estimaciones de las tasas relativas de mortalidad por deciles de la distribución del consumo de calorias se basaban en las estaturas medias estimadas y en el IMC de estos deciles y en los riesgos relativos de mortalidad que implican, como indica la figura 4. Para mayores detalles sobre los cálculos, véase el apéndice de Fogel y Floud (1994) y el cuadro A2 de Fogel (1993b).

41 David Seckler (1980), P. V. Sukhatme (1982), Michael Lipton (1983), cf. Patrick West et al. (1990).

42 Véase Fogel (1991), cf. Partha Dasgupta (1993). 
(medida en calorías), la mejora de la nutrición bruta explica alrededor de un 30 por ciento del crecimiento que experimentó la renta per cápita en Gran Bretaña entre 1790 y $1980^{43}$.

\section{B. Implicaciones para los países ricos}

Entre 1850 y 1950, la esperanza de vida al nacer aumentó en Estados Unidos de alrededor de 40 años a 68 . Durante las dos décadas siguientes, la longevidad apenas aumentó. Durante este interregno y el siguiente, los investigadores que examinaron los progresos de la mortalidad en el siglo anterior tendieron a coincidir en tres proposiciones:

(i) El descenso que registraron las tasas de mortalidad durante cien años fue único y no pudo repetirse porque se habían conseguido casi todas las mejoras logradas eliminando las muertes causadas por las enfermedades contagiosas por debajo de los 60 años.

(ii) Las muertes, concentradas ahora en las edades más avanzadas, se debian a enfermedades degenerativas que no estaban relacionadas con las enfermedades contagiosas a las que sustituyeron. Las enfermedades degenerativas se debian a pérdidas aceleradas de órganos que formaban parte del proceso natural de envejecimiento.

(iii) La esperanza de vida tenía un límite que era determinado genéticamente. Un influyente artículo lo situaba en $85 \pm 7$ años ${ }^{44}$.

Algunos estudios más recientes, que responden al nuevo descenso de la mortalidad, que en esta ocasión está concentrado en las personas de 65 años o más, han revelado datos que refutan la idea de que la esperanza de vida viene determinada genéticamente o, en caso de ser fija, sugieren que el límite máximo está por encima de los 85 años. El estudio de James W. Vaupel (1991a) sobre gemelos daneses indica que los factores genéticos sólo explican alrededor del 30 por ciento de la varianza de la edad al morir. Su estudio de los hombres suecos que vivían a los 90 años indica que la tasa de mortalidad a esa edad ha descendido a una tasa del orden de un 1 por ciento anual desde 1950 , resultado que es contradictorio con la rectagularización de la curva de supervivencia ${ }^{45}$. Dos estudios recientes de poblaciones de insectos indican

43 Fogel (1991).

44 James F. Fries (1980), cf. Fries (1989). (1994). 
que los cambios del medio ambiente influyen mucho más en la longevidad que los factores genéticos y no revelan la existencia de ninguna pauta que sugiera que habia un límite maximo fijo ${ }^{46}$. Considerados en su conjunto, estos estudios no excluyen los factores genéticos, pero sugieren algo mucho menos rígido que la programación genética de la longevidad absoluta. Está surgiendo una nueva teoria que combina la susceptibilidad genética de varios órganos con traumatismos acumulados causados por la exposición al riesgo.

Algunos estudios recientes también indican que las tasas de enfermedades crónicas por edades están disminuyendo generalmente en el caso de las personas mayores de 65 años. Según Manton et al. ${ }^{47}$, la tasa de incapacidad de los ancianos descendió un 4,7 por ciento entre 1982 y 1989 en Estados Unidos. Esta tasa de descenso, expresada en intervalos decenales, es bastante similar a las tasas de descenso a largo plazo de las enfermedades crónicas registradas entre 1910 y $1985-1988$ en el caso de los excombatientes de edad avanzada ${ }^{48}$. El resultado es coherente con las crecientes pruebas (publicadas en los apartados III y IV de este artículo) de que las enfermedades crónicas que padecen las personas de edad avanzada se deben en gran medida a la exposición a enfermedades infecciosas, a la desnutrición y a otros tipos de estrés biomédico y socioeconómico padecido en las primeras etapas de la vida. También es coherente con el descenso predicho de las enfermedades crónicas del orden del 6 por ciento por década basado en la superficie de Waaler correspondiente a la falta de salud mostrada en la figura $6^{49}$.

Actualmente, las investigaciones centran en su mayor parte la atención en la explicación del descenso de las enfermedades crónicas. Una parte de la explicación es el cambio del modo de vida, especialmente la reducción del consumo de tabaco, la mejora de la nutrición y el aumento del ejercicio, que parecen contribuir a reducir la incidencia de las enfermedades coronarias y respiratorias. Otra parte es el aumento de la eficacia de las intervenciones médicas. Este punto se demuestra sorprendentemente comparando la segunda columna de la línea sobre las hernias del cuadro 3 anterior con la última. Antes de la Segunda Guerra Mundial, las hernias generalmente eran permanentes una vez que aparecían y a menudo una enfermedad excesivamente dolorosa. Sin embargo, en la década de 1980 alrededor de tres cuartas partes de todos los excombatientes que habian tenido una hernia alguna vez estaban curados. La línea sobre las enfermedades genito-urinarias también indica que se han rea-

\footnotetext{
46 James R. Carey et al. (1992); James W. Curtsingere et al. (1992).

47 Manton et al. (1993).

48 Fogel et al. (1993).

49 Cf. Steven N. Blair et al. (1989); Manton et al. (1992); Manton y Beth J. Soldo (1992).
} 
lizado progresos similares durante las siete décadas. Otras áreas en las que las intervenciones médicas han sido sumamente eficaces son el control de la hipertensión y la reducción de la incidencia de la apoplejía, la eliminación quirúrgica de la osteoartritis, la sustitución de las articulaciones de la rodilla y la cadera, la curación de las cataratas y las quimioterapias que reducen la incidencia de la osteoporosis y las enfermedades de corazón ${ }^{50}$.

El éxito de las intervenciones médicas, unido al aumento de las rentas, ha dado lugar lógicamente a un enorme incremento de la demanda de servicios médicos. Las estimaciones econométricas sugieren que la elasticidad-renta a largo plazo de la demanda de servicios médicos en los países de la OCDE gira en torno a 1,5 e indican que el 90 por ciento de la varianza de los gastos médicos de los países de la OCDE se debe a las diferencias de renta ${ }^{51}$. El rápido crecimiento del nivel de demanda, unido a la política igualitaria de provisión de asistencia médica a precios muy subvencionados, ha provocado la crisis de los costes de la asistencia sanitaria que está siendo el centro de atención de los debates en todos los países de la OCDE, en los que está considerándose varias combinaciones de racionamiento basado en los precios e impuesto por los gobiernos 52 .

Cualquiera que sea el resultado final de estos debates, es evidente que nos encontramos en un mundo muy diferente al de Malthus. En lugar de debatir la conveniencia de suministrar alimentos a los pobres que, de lo contrario, podrian morir, estamos debatiendo cómo distribuir servicios que han conseguido aumentar la calidad de vida de los ancianos y prolongar la esperanza de vida. $Y$ actualmente estamos luchando con unas cuestiones éticas enteramente nuevas como la de si es correcto limitar los servicios médicos que prolongan vidas de baja calidad ${ }^{53}$.

El aumento de las oportunidades de mejorar la salud en las edades jóvenes, de reducir la incidencia de las enfermedades crónicas en las edades posteriores y de curar o aliviar las incapacidades relacionadas con enfermedades crónicas plantea otras dos cuestiones demográficas postmalthusianas. Una es la repercusión de la mejora de la salud en el volumen de población. Según un artículo reciente de Dennis A. Ahlburg y Vaupel ${ }^{54}$, si las tasas de mortalidad de las edades avanzadas continúan descendiendo un 2 por ciento al año, la pobla-

so Manton et al. (1993).

51 William J. Moore et al. (1992).

52 The Economist (1990), William B. Schwartz y Henry J. Aaron (1991), Joseph P. Newhouse (1992), George J. Schieber et al. (1993).

33 Barbara L. Wolfe (1986), John Sterling Shuttleworth (1990), Edmund D. Pellegrino (1993).

${ }_{44}$ Dennis A. Ahlburg y Vaupel (1990). 
ción anciana americana del año 2050 sería 36 millones mayor de lo que predice el Census Bureau ${ }^{55}$. Esa posibilidad plantea algunas cuestiones relacionadas con los costes de la asistencia sanitaria (ya que los costes médicos totales pueden aumentar acusadamente incluso aunque las tasas de curación continúen mejorando) y con los costes de las pensiones (ya que el número de personas que tienen derecho a percibir prestaciones según las normas propuestas actuales y los niveles previstos de indemnización serán tan altos que los pagos serán superiores a las reservas planeadas).

Algunos responsables de la política económica han tratado de resolver el problema de las pensiones retrasando la jubilación. Estos sistemas se basan en la proposición de que la mejora de la salud permitirá a más personas trabajar después de los 65 años. Sin embargo, los recientes resultados sobre la mejora secular de la salud a edades avanzadas muestran claramente que el empeoramiento de la salud no es la explicación del acusado descenso que han registrado desde 1890 las tasas de actividad de los varones mayores de 65 años. Como señala Costa ${ }^{56}$, el descenso de las tasas de actividad de los ancianos de la Unión que se ha registrado en los últimos cien años se debe en gran parte al aumento secular de la renta y a la disminución de la elasticidad-renta de la demanda de jubilación. También está relacionado con el enorme aumento de la oferta y la calidad de las actividades recreativas destinadas a las clases trabajadoras.

En tiempos de Malthus y hasta comienzos de siglo, la oferta de ocio era muy escasa en los paises de la OCDE y, como señala $T$. Veblen, era consumida claramente por una pequeña clase superior ${ }^{57}$. La persona representativa trabajaba más de 60 horas a la semana a cambio de un salario y muchas tenian que hacer labores domésticas que les llevaban otras 10 ó 12 horas más ${ }^{58}$. Además de dormir, comer y asearse, estos trabajadores normalmente apenas tenían dos horas de ocio al día. Aunque existia la ópera, el teatro y el ballet, éstos eran demasiado caros para que las clases trabajadoras pudieran consumirlos habitualmente.

En el siglo xx, las horas de trabajo han disminuido a casi la mitad en el caso de los trabajadores representativos. Paradójicamente, las personas pertenecientes al decil superior de la distribución de la renta no han participado mucho en este aumento del ocio, ya que los profesionales y empresarios bien

${ }^{5}$ Cf. Samuel H. Preston (1993).

56 Costa (1993).

57 T. Veblen (1934)

58 Kuznets (1952), John F. Olson (1992), Fogel (1993a), cf. Jeremy Atack y Fred Bateman (1992). 
remunerados que pueblan el decil superior trabajan un número de horas más próximo al patrón decimonónico de 3.200 horas al año que al de la clase trabajadora actual del orden de 1.800 horas. También ha aumentado inmensamente la oferta de ocio (películas, radio, televisión, parques de atracciones, espectáculos deportivos y viajes) y ha disminuido el precio relativo de esas actividades. Muchas empresas proporcionan servicios especialmente a los ancianos, ofreciendo precios reducidos y oportunidades especiales. Como consecuencia, el tiempo que dedica al ocio el trabajador representativo equivale a dos tercios del tiempo que dedica al trabajo y espera ansiosamente la jubilación 59.

Dado que la demanda de ocio existente en el mundo postmalthusiano de los paises de la OCDE es creciente e inelástica con respecto a la renta, queda por ver hasta qué punto puede asfixiarse la demanda de ocio y de jubilación. Los responsables de la política económica pueden encontrar tanta resistencia a los intentos de reducir las subvenciones implícitas al ocio como han encontrado recientemente para subir los impuestos sobre el trabajo.

\section{ALGUNAS IMPLICACIONES PARA LA TEORÍA Y LA MEDICIÓN DEL CRECIMIENTO ECONÓMICO}

Los recientes resultados obtenidos en el área biomédica llaman la atención sobre lo que podriamos denominar factores termodinámicos y fisiológicos del crecimiento económico. Aunque estos factores han sido dejados de lado en gran medida por los teóricos tanto de la «antigua» economía del crecimiento como de la «nueva», pueden incorporarse fácilmente a los modelos convencionales de crecimiento. Enfocados desde el punto de vista de capital humano, ambos factores pueden concebirse como cambios tecnológicos que aumentan el trabajo y que han sido provocados por acontecimientos ocurridos en el sector agrícola, en la sanidad pública, en los servicos médicos y en el sector de las economías domésticas. También pueden concebirse como ajustes para tener en cuenta los errores de medición de la cantidad trabajo, cuando ésta se mide solamente por el número de horas trabajadas.

Me he referido al factor termodinámico indirectamente en el apartado V-A cuando he indicado que alrededor de un 30 por ciento de la tasa de crecimiento que ha experimentado Gran Bretaña en los últimos 200 años se ha debido a la mejora de la nutrición bruta. Ese cálculo se basa en la primera ley de

${ }^{59}$ Fogel (1992a), (1993a). 
la termodinámica, según la cual la producción de energía no puede ser superior a la cantidad de energia utilizada como factor. Dado que la ley se aplica tanto a los motores humanos como a los mecánicos, es posible utilizar técnicas de contabilidad de los costes de la energía para estimar el aumento de la energía disponible para trabajar registrado en los dos últimos siglos. En el caso británico ese aumento ha producido dos efectos. Ha elevado la tasa de actividad al introducir en la población activa el 20 por ciento inferior de las unidades de consumo que en 1790 sólo tenían, en promedio, suficiente energía para deambular unas cuantas horas. Por otra parte, en el caso de las personas pertenecientes a la población activa, la intensidad del trabajo por hora ha aumentado debido a que ha aumentado el número de calorias disponibles para trabajar. Este cambio de la intensidad del esfuerzo parece que explica por sí solo alrededor de un 20 por ciento de la tasa de crecimiento a largo plazo.

Tal vez parezca ambiciosa la afirmación de que la intensidad del esfuerzo británico ha aumentado con el paso del tiempo, ya que la jornada, la semana y el año de trabajo (medidos en horas) han disminuido significativamente en los dos últimos siglos. Sin embargo, los británicos (y otros europeos) no podrían haber trabajado con la misma intensidad media por hora en 1790 que hoy, ya que para eso habrian necesitado una oferta de energia alimentaria per cápita muy superior a la que existía en realidad. Los aumentos de la intensidad de trabajo por hora también han sido importantes en el caso de la Unión, en el que las existencias de alimentos eran mucho más abundantes que en Europa. Incluso aunque se suponga que el número diario de calorias disponibles para trabajar era el mismo en Estados Unidos en 1860 que hoy, la intensidad del trabajo por hora habria sido muy inferior a la actual, ya que el número medio de horas trabajadas en 1860 era alrededor de 1,75 veces mayor que hoy. A mediados del siglo XIX, parece que los esclavos de las plantaciones sureñas basadas en el sistema de cuadrillas eran los únicos que trabajaban con un grado de intensidad por hora parecido al actual 60 .

El factor fisiológico está relacionado con la eficiencia con que el motor humano convierte la energía en trabajo. Los nutricionistas, los fisiólogos y los economistas del desarrollo han contribuido a la extensa literatura existente sobre este tema. Dado que algunas importantes cuestiones aún no se han resuelto, todavia no es posible hacer una valoración firme de la contribución fisiológica al crecimiento económico. Sin embargo, pueden indicarse algunos aspectos de esa contribución.

Los cambios de la salud, de la composición de la dieta y del vestido y el

${ }^{60}$ Cf. Fogel (1991), (1993a); Fogel y Stanley L. Engerman (1992), Olson (1992). 
alojamiento pueden influir significativamente en la eficiencia con que la energía ingerida se convierte en trabajo ${ }^{61}$. Las reducciones de la incidencia de las enfermedades infecciosas aumentan la proporción de energía ingerida disponible para trabajar, tanto por el ahorro de energía necesaria para movilizar el sistema inmunológico como porque mejora la capacidad del intestino para absorber los nutrientes, sobre todo como consecuencia de la reducción de las enfermedades diarreicas. La eficiencia termodinámica también ha aumentado gracias a los cambios de la composición de la dieta, incluida la sustitución de los cereales y otros alimentos que tienen un alto contenido de fibra por el azúcar y la carne. Estos cambios dietéticos han elevado la proporción de energía ingerida que puede metabolizarse (han aumentado el valor medio de los «factores de Atwater», por utilizar los términos de los nutricionistas). Las mejoras del vestido y el alojamiento también han aumentado la eficiencia termodinámica al reducir la cantidad de energía perdida a través de la radiación.

Los individuos que estén raquíticos pero, aparte de eso, estén sanos en la madurez correrán mayores riesgos de contraer enfermedades crónicas y de morir prematuramente. Para evaluar la importancia de las variaciones de la tasa de deterioro de la capacidad para trabajar durante el ciclo vital, es necesario calcular la influencia de los cambios de la estatura y del peso en el valor actual descontado de la diferencia entre las ganancias y los ingresos de subsistencia a lo largo del ciclo vital ${ }^{62}$. En las notas presentamos un procedimiento para estimar este efecto, así como estimaciones ilustrativas de las variables clave ${ }^{63}$. El

61 El análisis de este párrafo se basa en Dasgupta (1993) y en las fuentes en él citadas.

62 Cf. Dasgupta (1993).

63 El valor actual descontado del perfil de ganancias por edades de $n$ años a partir de la edad en que las ganancias alcanzan un máximo viene dado por

$$
P_{x}=E_{x} \int_{0}^{n} e^{-i(\mu+\pi) t} d t
$$

donde $\mu$ es la tasa de descenso de la función de supervivencia (la curva $l_{x}$ de la tabla de mortalidad), $\emptyset$ es la tasa de descenso de las ganancias netas anuales después de la edad $x, r$ es la tasa de descuento (que por razones de comodidad se supone que es igual a 6 por ciento), $x$ es la edad a la que las ganancias alcanzan un máximo, $E_{x}$ son las ganancias netas correspondientes a esa edad, $n$ es el número medio de años que transcurrieron entre $x$ y la edad media a la que un varón vivo dejaba de pertenecer con regularidad a la población activa (que, por razones de comodidad, se supone que es igual a 35) y $P_{x}$ es el valor actual descontado de la corriente de ganancias netas.

El valor de $\mu$ correspondiente a 1790 se ha calculado a partir de Wrigley y Schofield (1981) tomando la media de su $e_{0}$ correspondiente a $1786-1795$ (que es 36,63 ) e interpolando entre los niveles 8 y 9 de su familia de tablas de mortalidad inglesas para obtener la curva $l_{x}$ adecuada de alrededor de 1790 . El valor de $\mu$ en las edades $35-70$ de esa curva es 0,0289 .

El desplazamiento proyectado de la curva $l_{x}$ se basa en la superficie de Waaler del cuadro A1 de Fogel (1993b). Utilizando $1,68 \mathrm{~m}$ y $61 \mathrm{~kg}$. en el caso de 1790 y $1,76 \mathrm{~m}$. y $76 \mathrm{~kg}$. en el de 1980 , tenemos una predicción del descenso de la tasa de mortalidad del 31 por ciento. La curva $l_{x}$ correspondiente se ha obtenido a partir de las tablas Norte del modelo de Princeton corres- 
ejercicio indica que los ingresos descontados habrian aumentado alrededor de un 37 por ciento. Esta última cifra, unida a una conjetura sobre el efecto de los cambios de los factores de Atwater, sugiere que la eficiencia media del mo-

pondientes al nivel 14 de los varones, utilizando como base del ajuste $0,69\left({ }_{35} \mathrm{~m}_{35}\right)$. El valor de $\mu$ en las edades $35-70$ de esa curva $l_{x}$ es $-0,0202$.

Las variaciones de $E_{x}$ relacionadas con las variaciones de la estatura y el peso se han estimado a partir de una ecuación publicada por Robert A. Margo y Richard H. Steckel (1982). Los datos que utilizan se refieren a los esclavos capturados como botín de guerra por el ejército la Unión en 1863. Los tratantes que relacionaban el valor de los esclavos con su estatura y su peso parece que sólo se fijaban en las diferencias de localización (no de pendiente) del perfil de ganancias por edades, lo que indica que aparentemente no eran conscientes de la relación existente entre la estatura y el IMC, por una parte, y la mortalidad y las enfermedades crónicas, por otra (cf. Fogel, 1992c). La ecuación de Margo y Steckel es

$$
\begin{gathered}
\ln V=2,73+0,032 S+0,17 A-0,005 A^{2} \\
(1,47) \quad(0,92) \quad(2,22) \quad(-2,23) \\
+0,000046 A^{3}+0,053 H \\
(2,10) \quad(2,16) \\
+0,019 W-0,00027(H \times W) \\
(1,79) \quad(-1,73)
\end{gathered}
$$

$\left(N=523, R^{-2}=0,20\right)$, donde $V$ es el valor de un esclavo, $S$ es una variable ficticia correspondiente al color de la piel, $A$ es la edad, $H$ es la estatura (en pulgadas) y $W$ es el peso (en libras); los estadisticos $t$ se encuentran entre paréntesis. En el caso de 1790, he utilizado 66,1 pulgadas y 134 libras; en el de 1980, he utilizado 69,3 pulgadas y 167 libras. Estas cifras indican que $E_{x}$ aumentó alrededor de un 7 por ciento como consecuencia de los cambios relacionados con el tamaño del cuerpo.

El valor de $\emptyset$ correspondiente a 1790 en las edades 35-70 años se ha calculado a partir de los datos publicados en Fogel y Engerman (1974). Estos datos indican que las ganancias netas correspondientes a los 70 años eran alrededor de un 17 por ciento de las ganancias máximas, que se alcanzaban a los 35 años.

Con la información anterior y un supuesto inicial de que $\emptyset$ se mantuvo constante, el aumento de $P_{x}$ puede calcularse a partir de los datos mostrados en las ecuaciones (3) y (4):

$$
\begin{gathered}
P_{x, 1790}=\frac{E_{x}\left[1-e^{-00,0289+0,494+0,06135}\right]}{0,1383}=7,17 E_{x} \\
P_{x, p}=\frac{1,07 E_{x}\left[1-e^{-(0,0202+0,0494+0,06135}\right]}{0,1296}=8,17 E_{x}
\end{gathered}
$$

donde $P_{x, 1790}$ es el valor actual del perfil de ganancias de 1790 y $P_{x, p}$ es el valor actual del perfil proyectado a partir de las variaciones de la estatura y del IMC. Las ecuaciones (3) y (4) implican que $P_{x}$ aumentó un 14 por ciento $([8,17 / 7,17]-1=0,14)$.

Ahora es necesario tener en cuenta la influencia de los cambios del tamaño del cuerpo en la tasa de descenso de la función de ganancias netas (el valor de ø). Si se supone que las ganancias netas correspondientes a los 70 años aumentaron de 17 por ciento a 40 por ciento de las ganancias correspondientes a la edad de mayores ingresos como consecuencia de las mejoras fisiologicas, $P_{x}$ aumenta un 37 por ciento. Sin embargo, incluso esta última cifra probablemente sea demasiado baja, ya que no tiene en cuenta el desplazamiento secular del punto máximo del perfil de ganancias por edades de los 35 años aproximadamente hacia los 45 años. Por otra parte, los 
tor humano en Gran Bretaña aumentó alrededor de un 53 por ciento entre 1790 y 1980. El esfuerzo conjunto del aumento de la energía dietética disponible para trabajar y del aumento de la eficiencia humana en la transformación de la energía dietética en trabajo parece que ha representado alrededor de un 50 por ciento del crecimiento económico británico desde $1790{ }^{64}$.

Centrando la atención en los aspectos termodinámicos y fisiológicos del crecimiento económico, llaman la atención los largos desfases que se producen entre el momento en que se realizan determinadas inversiones y el momento en que se obtienen los beneficios. Una gran parte del aumento de la eficiencia termodinámica que se registró en Gran Bretaña y en otros países de la OCDE entre 1910 y 1980 se debió a una serie de inversiones realizadas nada menos que cien años antes. Si no se tienen en cuenta estos desfases extraordinariamente grandes entre las inversiones y los rendimientos, se observan enigmáticas paradojas. Por ejemplo, durante la Depresión de la década de 1930, la tasa de paro de Estados Unidos nunca fue inferior al 16 por ciento; durante la mitad de ese periodo, el paro osciló entre el 20 y el 25 por ciento. Sin embargo, la esperanza de vida aumentó en cuatro años entre 1929 y 1939 y la estatura de los hombres que llegaron a la madurez durante este periodo se elevó $1,6 \mathrm{~cm} .{ }^{65}$.

Creemos que la resolución de la paradoja se basa en las enormes inversiones sociales realizadas entre 1870 y 1930 , cuyos rendimientos no se contabilizaron en la renta nacional durante las décadas de 1920 y 1930, a pesar de que generaron una larga corriente de beneficios durante esas décadas. Nos referi-

estudios de los perfiles de los obreros de épocas recientes sugieren que actualmente las ganancias netas disminuyen mucho más despacio una vez sobrepasado el punto máximo que antiguamente (cf. Fogel y Engerman, 1974; Mary Jablonski et al., 1988; Kevin Murphy y Finis Welch, 1990).

${ }^{64}$ Se ha utilizado el siguiente procedimiento para obtener esta estimación: la entrada en la población activa del 20 por ciento inferior de la distribución calorica de 1790 aproximadamente aumentó la tasa de actividad un 25 por ciento. Por lo que se refiere a las personas pertenecientes a la población activa, el número medio de calorías disponibles para trabajar aumentó un 56 por ciento entre 1790 aproximadamente y 1980 . Por lo tanto, el incremento total de la producción per cápita generado por el aumento de la disponibilidad de calorias para trabajar fue de un 95 por ciento $(1,25 \times 1,56=1,95)$. El análisis de Dasgupta (1993) sugiere que las reducciones de las enfermedades diarreicas y de otros tipos, unidas a un cambio de la composición de la dieta, aumentó los factores de Atwater alrededor de un 12 por ciento. Dado que el ejercicio basado en la ecuación de la nota 8 implica que la reducción de las enfermedades crónicas y la mortalidad prematura aumentaron la eficiencia termodinamica un 37 por ciento, el aumento conjunto de la eficiencia termodinámica es del orden del 53 por ciento $(1,12 \times 1,37=1,53)$. Asi pues, en conjunto, el aumento de las calorias disponibles para trabajar y el aumento de la eficiencia termodinámica aumentaron la renta per cápita entre alrededor de 1870 y 1980 un 198 por ciento $(1,95 \times 1,53=2,98)$, o sea, un 9,58 por ciento al año $\left(2,98^{1 / 190}-1=0,0058\right)$, que es alrededor de la tasa anual británica de crecimiento $(0,58 / 1,15=0,50)$. Para mayores detalles, incluido un análisis de los posibles sesgos al alza y a la baja de este cálculo, véase Fogel (1987) y Fogel y Floud (1994).

65 Bernard D. Karpinos (1958), U. S. Bureau of the Census (1975). 
mos, por supuesto, a la inversión social en investigaciones biomédicas (incluido el establecimiento y la expansión de los hospitales modernos dedicados a la enseñanza y la investigación) cuyos mayores rendimientos se obtuvieron mucho después de que se realizó la inversión. En esta categoría también están incluidas algunas inversiones en saneamiento público, como la construcción de instalaciones para mejorar el suministro de agua, la depuración del suministro de leche, el drenaje de las ciénagas, el desarrollo de sistemas eficaces de cuarentena y la limpieza de los barrios pobres.

\section{COMENTARIOS FINALES}

Según Keynes, «a largo plazo todos estaremos muertos» ${ }^{66}$. Esta era una buena observación durante el periodo de entreguerras, en el que se registraron las graves inflaciones de los años 20 y la peor depresión de la historia durante los 30. Era necesario tomar medidas urgentes y enérgicas para recuperar el control de la oferta monetaria, atender a millones de parados e impedir el colapso de las democracias.

Vivimos en otra era. Las principales cuestiones que plantea la política económica en los paises de la OCDE en la actualidad no pueden comprenderse desde una mera perspectiva a corto plazo. La crisis de la asistencia médica, la crisis de las pensiones y los retos de la globalización se rigen por procesos a largo plazo que los responsables de la política económica deben comprender. Como hemos tratado de destacar en este artículo, aún no ha concluido la huida de la muerte por inanición y de la muerte prematura que comenzamos hace casi tres siglos. Aún sigue habiendo enfermedades crónicas y muertes prematuras incluso en los paises ricos. Para que tengan éxito las reformas de los programas de asistencia sanitaria y de pensiones que están considerando actualmente los responsables de la política económica, deben ser coherentes con los cambios fisiológicos a largo plazo que determinan la disminución de las enfermedades crónicas y el aumento de la longevidad. Las predicciones a largo plazo que no tienen en cuenta la dinámica de estos cambios ocurridos en el siglo pasado y de las mejoras socioeconómicas, biomédicas y otras mejoras del medio ambiente que los hicieron posible, corren el riesgo de no dar en el blanco.

Al comienzo de este artículo hemos subrayado la necesidad de que los economistas tengan en cuenta los procesos dinámicos a largo plazo estudiando la historia. El descubrimiento de lo que ocurrió realmente en el pasado requiere

66 Keynes (1971), p. 65. 
una enorme inversión de tiempo y esfuerzo. Afortunadamente para los teóricos, esa carga recae principalmente en los historiadores económicos. Los teóricos sólo necesitan dedicar el tiempo necesario a comprender lo que han descubierto los historiadores. Un conocimiento superficial de los trabajos de los historiadores económicos es, al menos, tan peligroso como un conocimiento superficial de la teoría.

\section{BIBLIOGRAFIAA}

Ahlburg, Dennis A., y Vaupel, James W. (1990): «Alternative Projections of the U. S. Population», Demography, 27(4), pp. 639-52.

ALLEN, R. C. (1994): «Agriculture during the Industrial Revolution, 1700-1850», en Roderick Floud y Donald McCloskey (eds.), The economic history of Britain since 1700, Cambridge, Cambridge University Press, $2^{a}$ ed.

Atack, Jeremy, y Bateman, Fred (1992): «How Long Was the Work Day in 1880?", Joumal of Economic History, 52(1), pp. 129-60.

BARKER, D. J. P. (1991): «The Intrauterine Environment and Adult Cardiovascular Di. sease", en Gregory R. Bock y Julie Whelan (eds.), The childhood environment and adult disease, Ciba Foundation Symposium 156, Chichester, Reino Unido, Wiley, pp. 3-16.

- (1993): «Fetal Origins of Coronary Heart Disease», British Heart Journal, 69(3), pp. $195-96$.

Barker, D. J. P.; Meade, T. W.; Fall, C. D. H.; Lee, A.; Osmond, C.; Phipps, K., y Stir LING, Y. (1992): «Relation of Fetal and Infant Growth to Plasma Fibrinogen and Factor VII Concentrations in Adult Life», British Medical Journal, 304(6820), pp. 148.52.

Barker, D. J. P.; Osmond, C., y Golding, J. (1990): «Height and Mortality in the Counties of England and Wales", Annals of Human Biology, 17(1), pp. 1-6.

Barker, D. J. P.; Osmond, C.; Golding, J.; Kuh, D., y Wadsworth, M. E. J. (1989): «Growth in Utero, Blood Pressure in Childhood and Adult Life, and Mortality from Cardiovascular Disease», British Medical Journal, (298(6673), pp. 564-67.

Bengtsson, Tommy, y Ohlsson, Rolf (1984): «Population and Economic Fluctuations in Sweden, 1749-1914», en Tommy Bengtsson, Gunnar Fridlizius y Rolf Ohlsson (eds.), Pre-industrial population change, Estocolmo, Almquist and Wiksell, pp. 277-97.

- (1985): «Age-Specific Mortality and Short-Term Changes in the Standard of Living: Sweden, 1751-1859», European Journal of Population, 1(4), pp. 309-26.

BILlewicz, W. Z., y MaCGREGORY, I. A. (1982): «A Birth to Maturity Longitudinal Study of Heights and Weights in Two West African (Gambian) Villages, 1951-1975», Annals of Human Biology, 9(4), pp. 309-20.

Blair, Steven N.; Kohl, Harold W., ILL.; Paffenbarger, Ralph S., Jr.; Clark, Debra G.; COOPER, Kenneth H., y GibBons, Larry W. (1989): Joumal of the American Medical Association, 262(17), pp. 2395-2401. 
Carey, James R.; Liedo, Pablo; Orozco, Dina, y Vaupel, James W. (1992): «Slowing of Mortality Rates at Older Ages in Large Medfly Cohorts", Science, 258(5081), pp. 457-61.

Chandra, R. K. (1975): «Antibody Formation in First and Second Generation Offspring of Nutritionally Deprived Rats» (1975), Science, 190(4211), pp. 289-90.

Chartres, J. A. (1985): "The Marketing of Agricultural Produce», en Joan Thirsk (comp.), The agrarian bistory of England and Wales, Cambridge, Cambridge University Press, vol. 5 (1640-1750, $2^{\mathrm{a}}$ Parte: Agrarian change), pp. 406-502.

Chavez, A.; Martinez, C., y Soberanes, B. (1994): «The Effect of Malnutrition on Human Development», en Nevin S. Scrimshaw (eds.), Longitudinal community based studies of the impact of early malnutrition on child bealth and development, Boston, MA, International Nutrition Foundation for Developing Countries.

Chen, Loncoln C.; Chowdhury, A. K. M. Alaudin, y Huffman, Sandra L. (1980): «Anthropometric Assessment of Energy-Protein Malnutrition and Subsequent Risk of Mortality Among Pre-School Aged Children», American Journal of Clinical Nutrition, 33(8), pp. 1836-45.

Cipolla, Carlo M. (1980): Before the industrial revolution: European society and economy, 1000-1700, Nueva York, Norton, $2^{*}$ ed.

Costa, Dora L. (1993): «Health, Income, and Retirement: Evidence from Nineteenth Century America", tesis doctoral, University of Chicago.

Curtsinger, James W; Fukui, Hidenori H.; Townsend, David R., y Vaupel, James W. (1992): «Demography of Genotypes: Failure of the Limited Life-Span Paradigm in Drosphila melanogaster, Science, 258(5081), pp. 461-63.

Dasgupta, Partha (1993): An inquiry into well-being and destitution, Oxford, Clarendon.

Dublin, Louis I., y LotKA, Alfred J. (1936): Lenght of life: $A$ study of the life table, Nueva York, Ronald.

Duncan, S. R.; ScotT, Susan, y Duncan, C. J. (1993): «The Dynamics of Smallpox Epjdemics in Britain, 1550-1800m, Demograpby, 30(3), pp. 405-23.

DupāquiER, Jacques (1989), «Demographic Crises and Subsistence Crises in France, 1650-1725», en John Walter y Roger Schofield (eds.), Famine, disease and the social order in early modern society, Cambridge, Cambridge University Press, pp. 189-99.

Eckstein, Zvi; Schultz, T. Paul, y Wolpin, Kenneth I. (1984): «Short-Run Fluctuations in Fertility and Mortality in Pre-industrial Sweden», European Economic Review, 26(3), pp. 297-317.

The Economist (1990), "Squeezing in the Next Five Billion», 20-26 de enero, 314(7638), pp. 19-20, 22.

FisLer, Janis S. (1992): «Cardiac Effects of Starvation and Semistarvation Diets: Safety and Mechanisms of Action», American Journal of Clinical Nutrition, julio, 56 (suplemento 1), pp. 230 S-34S.

FogEL, Robert Willliam (1986): «Nutrition and the Decline in Mortality Since 1700: Some Preliminary Findings», en Stanley L. Engerman y Robert E. Gallman (eds.), Long-term factors in American economic growth, Chicago, University of Chicago Press, pp. 439-555.

(1987): «Biomedical Approaches to the Estimation and Interpretation of Secular Trends in Equity, Morbidity, Mortality and Labor Productivity in Europe, 1750$1980 »$, manuscrito inédito, University of Chicago. 
(1991): «New Findings on Secular Trends in Nutrition and Mortality: Some Implications for Population Theory», manuscrito inédito, University of Chicago.

(1992a): «Egalitarianism: The Economic Revolution of the Twentieth Century», The 1992 Simon Kuznets Memorial Lectures presentadas en Yale University, 24-24 de abril.

(1992b): «Second Thoughts on the European Escape from Hunger: Famines, Chronic Malnutrition, and Mortality», en S. R. Osmani (eds.), Nutrition and poverty, Oxford, Clarendon, pp. 243-86.

(1992c): «The Body Mass Index of Adult Male Slaves in the U. S. c. 1863 and Its Bearing on Mortality Rates», en Robert William Fogel, Ralph A. Galantine y Richard L. Manning (eds.), Without consent or contract, Nueva York, Norton, vol. 2 (Evidence and methods), pp. 311-18.

- (1993a): «a Comparison of Biomedical and Economic Measures of Egalitarianism: some Implications of Secular Trends for Current Policy», manuscrito inédito presentado en el Workshop on Economic Theories of Inequality, Stanford University, 11-13 de marzo.

- (1993b): «New Sources and New Techniques for the Study of Secular Trends in Nutritional Status, Health, Mortality, and the Process of Aging», Historical Metbods, 26(1), pp. 5-43.

Fogel, Robert William; Costa, Dora L., y KIM, John M. (1993): «Secular Trends in the Distribution of Chronic Conditions and Disabilities at Young Adult and Late Ages, 1860-1988: Some Preliminary Findings", manuscrito inédito presentado en el NBER Summers Institute, Economics of Aging Program, 26-28 de julio.

Fogel, Robert William, y EngERman, Stanley L. (1974): Time on the cross: The Economics of American Slavery, Boston, Little, Brown.

(1992): «The Slave Diet on Large Plantations in 1860», en Robert William Fogel, Ralph A. Galantine y Richard L. Manning (eds.), Without consent or contract, Nueva York, Norton, vol. 2 (Evidence and methods), pp. 291-304.

Fogel, Robert William, y Floud, Roderick (1994): «Nutrition and Mortality in France, Britain, and the United Statesm, manuscrito inédito, University of Chicago.

Freudenberger, Herman, y Cummins, Gaylord (1976): «Health, Work, and Leisure Before the Industrial Revolution», Explorations in Economic History, 13(1), pp. 1-12.

Fridlizius, Gunnar (1984): «The Mortality Decline in the First Phase of the Demographic Transition: Swedish Experiences», en Tommy Bengtsson, Gunnar Fridlizius y Rolf Ohlsson (eds.), Pre-industrial population change, Estocolmo, Almquist and Wiksell, pp. 71-114.

Friedman, Gerald C. (1982): «The Heights of Slaves in Trinidad», Social Science History, 6(4), pp. 482-515.

FrIES, James F. (1980): «Aging, Natural Death, and the Compression of Morbidity», New England Journal of Medicine, 303(3), pp. 130-36.

- (1989): «The Compression of Morbidity: Near or Far?», Milbank Quarterly, 67(2), pp. 208-32.

Galloway, Patrick R. (1986): «Differentials in Demographic Responses to Annual Price Variations in Pre-Revolutionary France: A Comparison of Rich and Poor Areas in Rouen, 1681-1787», European Journal of Population, 2(3-4), pp. 269-305.

(1987): «Population, Prices and Weather in Preindustrial Europe», tesis doctoral, University of California, Berkeley. 
Gille, H. (1949): «The Demographic History of Northern European Countries in the Eighteenth Century», Population Studies, pp. 3-70.

Goubert, Pierre (1973): The ancien régime, Nueva York, Harper, trad. de Steven Cox.

Gould, Benjamin Apthorp (1989): Investigations in the military and antbropological statistics of American soldiers, Nueva York, Hurd and Houghton.

HeYwood, Peter F. (1983): «Growth and Nutrition in Papua New Guinea», Journal of Human Evolution, 12(1), pp. 131-43.

Hill, G. L. (1990): «Some Implications of Body Composition Research for Modern Clinical Management», Infusionstherapie, abril, 17 (suplemento 3), pp. 79-80.

Holderness, B. A. (1989): «Prices, Productivity, and Output», en G. E. Mingay (eds.), The agrarian bistory of England and Wales, Cambridge, Cambridge University Press, vol. 6 (1750-1850), pp. 84-189.

HyTten, F. E., y LeITCH, I. (1971): The pbysiology of human pregmancy, Oxford, Blackwell, $2^{*} \mathrm{ed}$.

IdiAquez, Juan (1988): «Nutritional Status and Autonomic Nervous System Function», Functional Neurology, abril-junio, 3(2), pp. $205-9$.

Jablonski, Mary; Rosenblum, Larry, y KunZe, Kent (1988): «Productivity, Age, and Labor Composition Changes in the U. S.», Montbly Labor Review, septiembre, 111(9), pp. 202-29.

JoHN, A. Meredith (1988): The plantation slaves of Trinidad, 1783-1816. A mathematical and demographic inquiry, Cambridge, Cambridge University Press.

Kannisto, V.; Lauritsen, J.; Thatcher, A. R., y Vaupel, J. W. (1994): «Reductions in Mortality at Advances Ages», Population and Development Review.

KarPINOS, Bernard D. (1958): «Height and Weight of Selective Service Registrants Processed for Military Service during WW II», Human Biology, 30(4), pp. 292-321.

KEYNES, John Maynard (1971 [1923]): Collected writings of Jobn Maynard Keynes, Londres, Macmillan, vol. 4 (A Tract on Monetary Reform).

Kielmann, Arnfried A.; Desweemer, Cecile; Chernichovsky, Dov; Uberoi, Inder S.; MA. SIH, Norah; Taylor, Carl E.; PARKer, Robert L.; Reinke, William A.; KaKar, D. N., y SARMA, R. S. S. (1983): Cbild and maternal bealth services in rural India: The Narangwal experiment, Baltimore, MD, Johns Hopkins University Press, vol. 1 (Integrated nutrition and bealth).

KIM, John M. (1993): «Economic and Biomedical Implications of Waaler Surfaces: A New Perspective on Height, Weight, Morbidity, and Mortality», manuscrito inédito, University of Chicago.

KuZnETS, Simon (1941): «Statistics and Economic History», Joumal of Economic History, mayo, 1(1), pp. 26-41.

(1952): «Long-Term Changes in the National Income of the United States of America Since 1879", en Simon Kuznets (eds.), Income and wealth of the United States: Trends and structure, International Association for Research in Income and Wealth, Income and Wealth Series 2, Baltimore, MD, Johns Hopkins University Press, pp. 2-241.

LASLETT, Peter (1984): The world we bave lost: England before the industrial age, Nueva York, Scribner's, $3^{\text {a }}$ ed.

Law, C. M.; De Swiet, M.; Osmond, C.; Fayers, P. M.; Barker, D. J. P.; Cruddas, A. M., y FALL, C. H. D.: "Initiation of Hypertension in Utero and Its Amplification Throughout Life», British Medical Joumal, 2 de enero, 306(6869), pp. 24-27. 
LEE, Ronald (1981): «Short-Term Variation: Vital Rates, Prices, and Weather», en E. A. Wrigley y R. S. Schofield (eds.), The population bistory of England, 1541-1871: A reconstruction, Cambridge, MA, Harvard University Press, pp. 356-401.

LIPTON, Michael (1983): «Poverty, Undernutrition and Hunger», Banco Mundial, Washington D. C., Staff Working Paper n. 597.

Livi-Bacci, Massimo (1990): Population and nutrition: An essay on European demographic bistory, Nueva York, Cambridge University Press.

Lozoff, Betsy; JiménEz, Elías, y WOLF, Abraham W. (1991): «Long-Term Developmental Outcome of Infants with Iron Deficiency», New England Journal of Medicine, $325(10)$, pp. $687-95$.

Manton, Kenneth G. (1993): «Biomedical Research and Changing Concepts of Disease and Aging: Implications for Long-Term Forecasts for Elderly Populations», en Kenneth G. Manton, Burton H. Singer y Richard M. Suzman (eds.), Forecasting the bealt $b$ of elderly populations, Nueva York, Springer-Verlag, pp. 319-65.

Manton, Kenneth G.; Corder, Larry S., y Stallard, Eric (1993): «Estimates of Change in Chronic Disability and Institutional Incidence and Prevalence Rates in the U.S. Elderly Population from the 1982, 1984, and 1989 National Long-Term Care Survey», multicopiado, Center for Demographic Studies, Duke University.

Manton, Kenneth G., y Soldo, Beth J. (1992): «Disability and Mortality among the Ol. dest Old: Implications for Current and Future Health and Long-Term Care Service Needs», en Richard M. Suzman, Kenneth G. Manton y David P. Willis (eds.), The oldest old, Nueva York, Oxford University Press, pp. 199-250.

Manton, Kenneth G.; STallard, Eric, y Singer, Burt (1992): «Projecting the Future Size and Health Status of the U. S. Elderly Population», International Joumal of Forecasting, 8(3), pp. 433-58.

MARGO, Robert A., y STECKEL, Richard H. (1982): «The Heights of American Slaves: New Evidence on Slave Nutrition and Health», Social Science History, 6(4), pp. 516-38.

Marmot, M. G.; Shipley, M. J., y Rose, Geoffrey (1984): «Inequalities in Death-Specific Explanations of a General Pattern?», Lancet (8384), pp. 1003-6.

Martorell, R. (1985): «Child Growth Retardation: A Discussion of Its Causes and Its Relationship to Health», en Sir Kenneth Blaxter y J. C. Waterlow (eds.), Nutritional adaptation in man, Londres, Libby, pp. 13-29.

Martorell, R.; Rivera, J., y Kaplowitz, H. (1990): «Consequences of Stunting in Early Childhood for Adult Body Size in Rural Guatemala», Annales Nestlé, 48, pp. 85-92.

Mcmahon, M. Molly, y Bistrian, Bruce R. (1990): «The Physiology of Nutritional Assessment and Therapy in Protein-Calorie Malnutritionm, Disease-a-Month 36(7), pp. 373-417.

Megrton, M. A. von (1989): «Croissance économique en France et accroissement des Française: Une analyse 'Villermetrique'», manuscrito inédito, Center voor Economische Studiën, Lovaina, Bélgica.

Moore, William J.; Newman, Robert J., y Fheili, Mohammad (1992): «Measuring the Relationship between Income and NHEs», Health Care Financing Review, otoño, 14(1), pp. 133-39.

Murphy, Kevin M., y Welch, Finis (1990): «Empirical Age-Earnings Profiles», Joumal of Labor Economics, abril, 8(2), pp. 202-29. 
Newhouse, Joseph P. (1992); «Medical Care Costs: How Much Welfare Loss?», Journal of Economic Perspectives, 6(3), pp. 3-21.

OdDY, D. J. (1990): «Food, Drink and Nutrition», en F. M. L. Thompson (comp.), The Cambridge social history of Britain, 1750-1950, Nueva York, Cambridge University Press, vol. 2 (People and their environment), Cambridge University Pres, pp. 251-78.

Olson, John F. (1992): «Clock Time versus Real Time: A Comparison of the Lenghts of the Northern and Southern Agricultural Work Years», en Robert William Fogel y Stanley L. Engerman (eds.), Without consent or contract, Nueva York, Norton, vol. 3 (Markets and production: Technical Papers, vol. 1), pp. 216-40.

Organizacion para la Agricultura $Y$ la Alimentación, Organizacion Mundial de la SALUd Y UNIVERSIDAD DE LAS NACIONES UNIDAS (1985): Energy and protein requirements. Report of a joint FAO/WHO/UNU expert consultation, Technical Report Series n. ${ }^{\circ} 724$, Ginebra, Organización Mundial de la Salud.

Osmani, S. R. (1992): «On Some Controversies in the Measurement of Undernutrition», en S. R. Osmani (comp.), Nutrition and poverty, Oxford, Clarendon, pp. 121-64.

PAYNe, P. (1992): «Assessing Undernutrition: The Need for a Reconceptualization», en S. R. Osmani (comp.), Nutrition and poverty, Oxford, Clarendon, pp. 49-96.

Pellegrino, Edmund D. (1993): «The Metamorphosis of Medical Ethics: A 30-Year Retrospective», Joumal of the American Medical Association, 269(9), pp. 1158-62.

Perrenoud, Alfred (1984): «The Mortality Decline in a Long-Term Perspective», en Tommy Bengtsson, Gunnar Fridlizius y Rolf Ohisson (eds.), Pre-industrial popuLation change, Estocolmo, Almquist and Wiksell, pp. 41-69.

(1991): «The Attenuation of Mortality Crises and the Decline of Mortality», en R. Schofield, D. Reher y A. Bideau (eds.), The decline of mortality in Europe, Oxford, Clarendon, pp. 18-37.

Phillips, D. I. W.; Cooper, C.; Fall, C.; Prentice, L.; Osmond, C.; Barker, D. J. P., y ReEs, Smith B. (1993): «Fetal Growth and Autoimmune Thyroid Disease», Quarterly Joumal of Medicine 86(4), pp. 247-53.

Phipps, K.; Barker, D. J. P.; Hales, C. N.; Fall, C. H. D.; Osmond, C., y Clark, P. M. S. (1993): «Fetal Growth and Impaired Glucose Tolerance in Men and Women», Diabetologia, marzo, 36(3), pp. 225-28.

Preston, Samuel H. (1993): «Demographic Changes in the United States, 1970-2050», en Anna M. Rappaport y Sylvester J. Schieber (eds.), Demography and retirement: The twenty-first century, Westport, CT, Praeger, pp. 19-48.

Quenouille, M. H.; Boyne, A. W.; Fisher, W. B., y Leitch, I. (1951): Statistical studies of recorded energy expenditure in man, Technical Communication n..$^{\circ} 17$, Aberdeenshire, Escocia, Commonwealth Bureau of Animal Nutrition.

Richards, Toni (1984): «Weather, Nutrition and the Economy: The Analysis of Short Run Fluctuations in Births, Deaths and Marriages, France 1740-1909», en Tomomy Bengtsson, Gunnar Fridlizius y Rolf Ohlsson (eds.), Pre-industrial population change, Estocolmo, Almquist and Wiksell, pp. 357-89.

Robbins, S. L.; Cotran, R. S., y Kumar, V. (1984): Pathologic basic of disease, Filadelfia, Saunders, $3^{\mathrm{a}}$ ed.

SABA, Thomas M.; Dillon, Bruce C., y Lanser, Marc E. (1983): «Fibronectin and Phagocytic Host Defense: Relationship to Nutritional Support», Joumal of Parenteral and Enteral Nutrition, 7(1), pp. 62-68. 
SChieber, George J.; Pouldier, Jean-Pierre, y Greenwald, Leslie M. (1993): «Health Care Systems in Twenty-Four Countries», Healtb Affairs, 10(3), pp. 22-38.

Schwartz, William B., y Aaron, Henry J. (1991): «Must We Ration Health Care?», Best's Review, enero, 91(9), pp. 37-41.

SCrimshaw, Nevin S. (1993): «Malnutrition, Brain Development, Learning and Behavior», The Twentieth Kamla Puri Sabharwal Memorial Lecture presentada en el Lady Irwin College, Nueva Delhi, 23 de noviembre.

SCrimshaw, Nevin S., y Gordon, J. E. (eds.) (1968): Malnutrition, learning and behavior, Cambridge, MA, MIT Press.

SCrimshaw, Nevin, S.; TAYlOR, C. E., y Gordon, J. E. (1968): Interactions of nutrition and infection, Ginebra, Organización Mundial de la Salud.

SECKLER, David (1980): “Malnutrition': An Intellectual Odyssey», Western Joumal of Agricultural Economics, 5(2), pp. 219-27.

SEN, Amartya (1981): Poverty and famines: An essay on entitlement and deprivation, Oxford, Clarendon.

Shammas, Carole (1984): «The Eighteenth-Century English Diet and Economic Change», Explorations in Economic History, 21(3), pp. 254-69.

- (1990): The pre-industrial consumer in England and America, Oxford, Clarendon.

ShuttleworTh, John Sterling (1990): «Ethical Issues in Long-Term Care», Journal of the Medical Association of Georgia, 79(11), pp. 843-45.

Sommer, Alfred, y Lowenstein, Matthew S. (1975): «Nutritional Status and Mortality: A Prospective Validation of the QUAC Stick», American Journal of Clinical Nutrition, 28(3), pp. 287-92.

SRInivasan, T. N. (1992): «Undernutrition: Concepts, Measurement, and Policy Implications», en S. R. Osmani (comp.), Nutrition and poverty, Oxford, Clarendon, pp. 97-120.

Sukhatme, P. V. (1982): «Poverty and Malnutrition», en P. V. Sukhatme (eds.), Newer concepts in Nutrition and their implications for policy, Pune, India, Maharashtra Association for the Cultivation of Science Research Institute, pp. 11-63.

TANNER, J. M. (1990): Foetus into man: Pbysical growth from conception to maturity, ed. rev., Cambridge, MA, Harvard University Press.

- (1993): Reseña de D. J. P. Barker, Fetal and infant origins of adult disease. Annals of Human Biology, 20(5), pp. 508-9.

Thatcher, A. R. (1992): «Trends in Numbers and Mortality at High Ages in England and Wales», Population Studies, 46(3), pp. 411-26.

Toutain, Jean-Claude (1971): «La consommation alimentaire en France de 1789 à 1964», Economies et Sociétés, Cabiers de lTSEA, 5(11), pp. 1909-2049.

U. S. BuREAU OF THE Census (1975): Historical statistics of the United States, colonial times to 1970, Washington, D. C., U. S. Government Printing Office.

U. S. Department of Health and Human Series (1987): Anthropometric Reference Data and Prevalence of Overweight, Vital and Health Statistics, Series 11 n. ${ }^{\circ} 238$, Washington, D. C., U. S. Government Printing Office.

VAUPEL, James W. (1991a): «The Impact of Population Aging on Health and Health Care Costs: Uncertainties and New Evidence About Life Expectancy», manuscrito inédito, Center for Health and Social Policy, Odense University, Dinamarca.

(1991): «Prospects for a Longer Life Expectancy», manuscrito inédito presentado en la reunión anual de la Population Association of America, Washington, D. C., 21.23 de marzo. 
VAUPEL, James W., y LundSTROM, H. (1994): «Prospects for Longer Life Expectancy», en David Wise (comp.), Economics of aging, Chicago, University of Chicago Press.

VEBLEN, T. (1934 [1899]): The theory of the leisure class: An economic study of institutions, Nueva York, Modern Library.

VOLPE, J. J. (1987): «Hypoxic-Ischemic Encephalopathy-Clinical Aspects», en Neurology of the newborn, Filadelfia, Saunders, $2^{a}$ ed., pp. 236-79.

WAALER, Hans Th. (1984): «Weight, Weight and Mortality: The Norwegian Experience», Acta Medica Scandinavica, suplemento (679), pp. 1-51.

WEIR, David R. (1982): «Fertility Transition in Rural France, 1740-1829", tesis doctoral, Stanford University.

(1989): «Markets and Mortality in France, 1600-1789», en John Walter y Roger Schofield (eds.), Famine, disease and the social order in early modern society, Cambridge, Cambridge University Press, pp. 201-34.

(1993): «Parental Consumption Decisions and Child Health during the Early French Fertility Decline, 1790-1914», Journal of Economic History, 53(2), pp. 259-74.

West, Patrick; Macintyre, Sally; Annandale, Ellen, y Hunt, Kate (1990): «Social Class and Health in Youth: Findings from The West of Scotland Twenty-O7 Study", Social Science and Medicine, 30(6), pp. 665-73.

WolfE, Barbara, L. (1986): «Health Status and Medical Expenditures: Is There a Link?», Social Science and Medicine, 22(10), pp. 993-99.

WRIGLEY, E. A. (1987): «Urban Growth and Agricultural Change: England and the Continent in the Early Modern Period», en Peoples, cities and wealth: The transformation of traditional society, Oxford, Blackwell, pp. 157-93.

WRIGLEY, E. A., y SCHOFIELD, R. S. (eds.) (1981): The population bistory of England, 1541-1871: A reconstruction, Cambridge, MA, Harvard University Press.

Traducción de Estber Rabasco y Luis Tobaria. 